\title{
A Lower Extremity Exoskeleton: Human-Machine Coupled Modeling, Robust Control Design, Simulation, and Overload-Carrying Experiment
}

\author{
Qing Guo, ${ }^{1}$ Songjing $\mathrm{Li}^{2}{ }^{2}$ and Dan Jiang ${ }^{3}$ \\ ${ }^{1}$ School of Aeronautics and Astronautics, University of Electronic Science and Technology of China, Chengdu 611731, China \\ ${ }^{2}$ Department of Fluid Control \& Automation, Harbin Institute of Technology, Harbin 150001, China \\ ${ }^{3}$ School of Mechatronics Engineering, University of Electronic Science and Technology of China, Chengdu 611731, China \\ Correspondence should be addressed to Qing Guo; guoqinguestc@163.com
}

Received 4 November 2014; Revised 8 May 2015; Accepted 10 May 2015

Academic Editor: Stanisław Migorski

Copyright (C) 2015 Qing Guo et al. This is an open access article distributed under the Creative Commons Attribution License, which permits unrestricted use, distribution, and reproduction in any medium, provided the original work is properly cited.

\begin{abstract}
A robust $H_{\infty}$ control method and switched control algorithm for hydraulic actuator presents in human-machine coordinated motion to solve the motion delay of lower extremity exoskeleton. After the characteristic parameters synthesis of human limb and exoskeleton linkage, the human-machine coupled motion model is constructed to estimate the appropriate hydraulic pressure, which is considered as a structural uncertainty in hydraulic model. Then the robust controller is designed to improve the robust stability and performance under the structural and parametric uncertainty disturbances. Simulation results show that, in walking mode, this robust controller can achieve a better dynamic response and aid-force efficiency than PID controller. Then, according to gait divisions of person's limb motion, the switched control algorithm is designed to reduce the delay of exoskeleton tracking person. Finally, the experimental results show that the human-machine coordinated walk with bearing $60 \mathrm{~kg}$ load and squat action with no external load are realized effectively by this proposed method.
\end{abstract}

\section{Introduction}

Lower extremity exoskeleton is a humanoid mechanical equipment. Since the exoskeleton can help a person bear many loads and track a person's motion, the soldier's load capacity and flexibility can be significantly improved. The exoskeleton is a complex system including 4 subsystems: mechanical structure, gait sensing, hydraulic control, and power supply. Recently lower extremity exoskeleton can bear the maximum load $200 \mathrm{lbs}$ and realize typical action such as walk, running, up and down hill or stairs, squatting, and trotting [1]. Since 2000, DARPA and U. C. Berkeley have done many researches on exoskeleton and developed the prototypes called "XOS" and "BLEEX" [2-4], respectively. Lockheed has simplified the hydraulic actuator amount of BLEEX and renamed "Human Universal Load Carrier" (HULC) [1]. Other countries like Japan and France have developed commercial or military exoskeleton called "HAL" or "Hercule" [5]. In 2000, Atkeson et al. [6] made conclusions on the characteristics of humanoid robot: sometimes the motion of machine is guided by person, sometimes just the opposite, especially sometimes the boundary is not clearly divided between the two. Due to the person's participation, the traditional robotic program control method will cause the motional delay of exoskeleton tracking person [7].

Since 2004, the exoskeleton technology is developed in China [8]. A motor-driven exoskeleton with no external load is designed by Naval Aeronautical Engineering Institute [9]. In addition, the aid-force exoskeleton with hydraulic-driven actuator is designed to realize walk, up and down stairs [10]. However, the human-machine coordinated motion is not presented in aforementioned references; if person's gait characteristic is ignored in exoskeleton control, person's comfort and the aid-force effect are reduced. In this paper, a human-machine coupled model is constructed to analyze the dynamic external load. This external load describes the aidforce efficiency of exoskeleton in the case of person's safety and should be compensated by exoskeleton controller. 
Due to the demands of high load efficiency and fast response, lower extremity exoskeleton is driven by the electrohydraulic servo system (EHSS). However, EHSS has some model uncertainties such as parametric disturbances, structural uncertainties, which are consequences of hydraulic physical characteristics, oil leakage, oil temperature variations, and characteristics of valves near null [11]. The classical control method of EHSS is PID [12]. Due to lower order and constant gains, PID control is easy to be realized in engineering. So the variable parameters of PID controller are presented to suit the hydraulic dynamic characteristic $[13,14]$. Some advanced control methods such as adaptive sliding mode [15-17] and backstepping controllers [18, 19] are used in EHSS and get some good performance. In addition, the robust controllers [20-22] of EHSS are presented with parameter uncertainty disturbance, which are converted to $H_{\infty}$ control problem solved by conventional robust analysis or linear matrix inequality. The mixed sensitivity $H_{\infty}$ control method [23] of EHSS is presented to balance to the robust stability and response performance. But the external load of hydraulic control model is only considered as a disturbance, not to be quantitatively analyzed and described in robust uncertainty model. In this paper, by referring to $H_{\infty}$ control method aforementioned references, a robust $H_{\infty}$ controller is designed to suppress structural uncertainty and parametric uncertainties, which are caused by the external load and parametric disturbances. Furthermore, combined with the switched control algorithm of hydraulic actuator, this $H_{\infty}$ controller can reduce the delay of exoskeleton tracking person. Simulation and experimental results validate this proposed controller.

\section{Human-Machine Coupled Model Construction}

Since human fast conscious is ahead of exoskeleton, the only mechanical motion model is not insufficient to analyze the systematic dynamics. So the coupled motion model needs to be constructed to estimate the dynamic loads and the supply power.

2.1. Characteristic Parameters Synthesis. Some characteristic parameters like mass and moment of inertia are different between human limb and exoskeleton linkage. So the characteristic parameters need be synthetic to balance 2 motion responses activated by person and exoskeleton. It means that human-constraint is relieved but not vanished through equivalent multibody motion. The human-constraint is a small value in the thigh bandage, which can not exceed the human tolerance.

If the sagittal motion is considered only, the rotational motion around the knee is shown in Figure 1. Here components $B_{1}, B_{2}, B_{3}, B_{4}$, and $B_{5}$ represent, respectively, human calf, mechanical calf, sensing shoes, human thigh, and mechanical thigh.

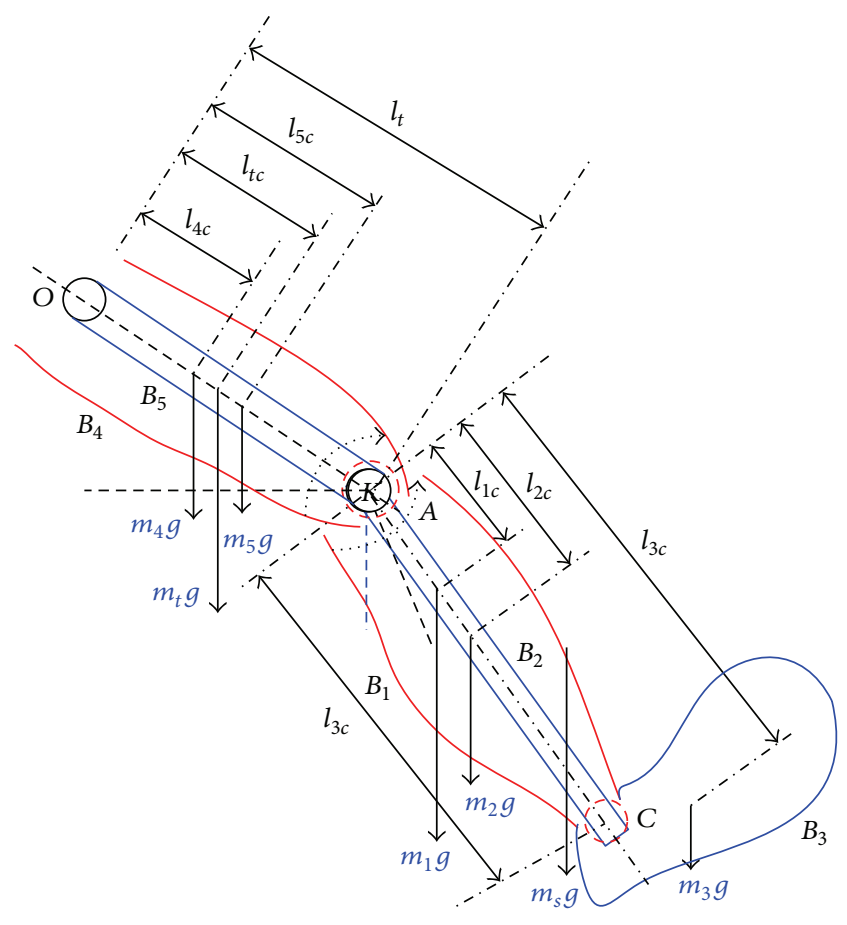

FIGURE 1: The rotational motion around the knee of human-machine synthetic model.

The centers of synthetic calf and thigh for humanmachine coordinated motion [24] are shown as

$$
\begin{aligned}
& l_{s c}=\frac{m_{1} l_{1 c}+m_{2} l_{2 c}+m_{3} l_{3 c}}{m_{1}+m_{2}+m_{3}}=\frac{m_{1} l_{1 c}+m_{2} l_{2 c}+m_{3} l_{3 c}}{m_{s}}, \\
& l_{t c}=\frac{m_{4} l_{4 c}+m_{5} l_{5 c}}{m_{4}+m_{5}}=\frac{m_{4} l_{4 c}+m_{5} l_{5 c}}{m_{t}} .
\end{aligned}
$$

According to the moment of inertia of the parallel axis theorem, the moment of inertia of synthetic calf and thigh around respective center of mass is shown as

$$
\begin{aligned}
I_{s c}= & {\left[I_{1 c}+m_{1}\left(l_{s c}-l_{1 c}\right)^{2}\right]+\left[I_{2 c}+m_{2}\left(l_{s c}-l_{2 c}\right)^{2}\right] } \\
& +\left[I_{3 c}+m_{3}\left(l_{s c}-l_{3 c}\right)^{2}\right], \\
I_{t c}= & {\left[I_{4 c}+m_{4}\left(l_{t c}-l_{4 c}\right)^{2}\right]+\left[I_{5 c}+m_{5}\left(l_{t c}-l_{5 c}\right)^{2}\right], }
\end{aligned}
$$

where $m_{i}$ is the $i$ th component mass. $m_{s}$ and $m_{t}$ are the total masses of equivalent calf and thigh, $l_{1 c}, l_{2 c}$, and $l_{3 c}$ are the distances from center of mass to knee, respectively, $l_{4 c}, l_{5 c}$ are the distances from center of mass to hip, respectively, and $I_{i c}$ is the $i$ th moment of inertia around respective center of mass.

2.2. Human-Machine Coupled Motion Model. In humanmachine coordinated motion, the exoskeleton linkages should compensate the load when human foot contacts the ground. So in the stance phase of walk or squat, the hydraulic actuator not only realizes the exoskeleton knee rotation tracking human limb rotation but also balances the variable load. In order to estimate the dynamic load on the hydraulic 


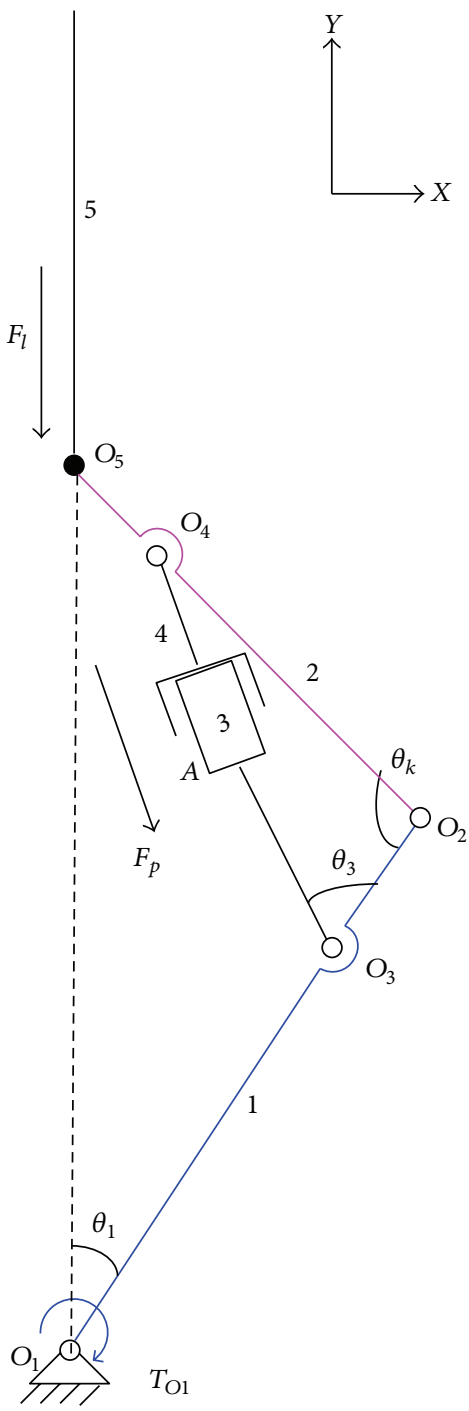

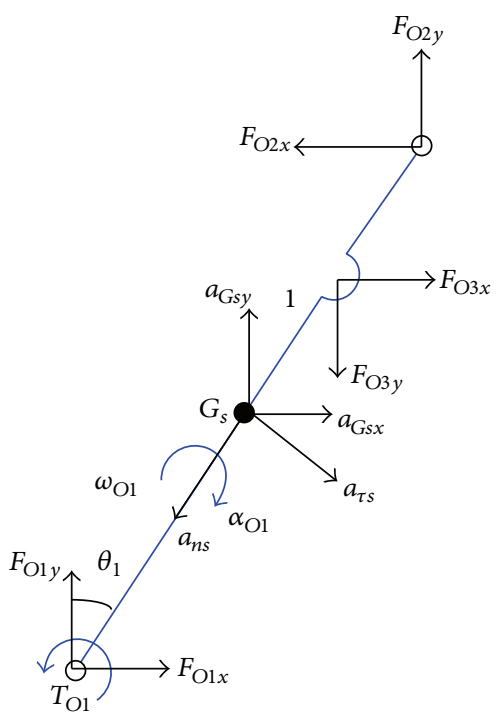

(a)

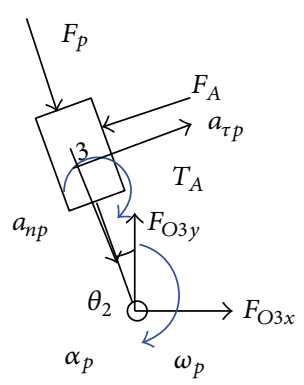

(c)

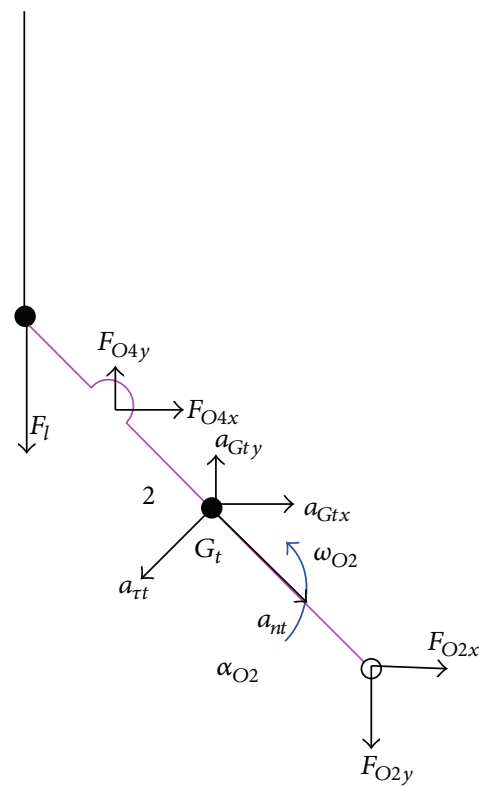

(b)

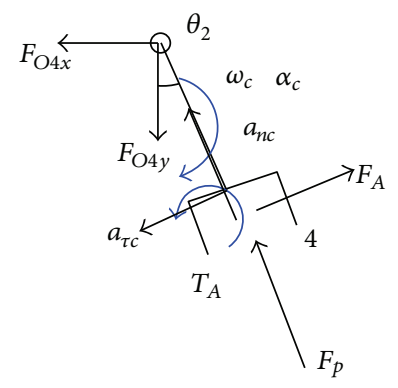

(d)

FIGURE 2: The synthetic model of human-machine coupled motion (linkages 1 and 2 represented the synthetic calf and thigh).

actuator, the human-machine coupled motion model needs to be constructed in Figure 2. The link point " $\mathrm{O}_{5}$ " between mechanical back frame and thigh is the hip joint. The ankle joint " $\mathrm{O}_{1}$ " is equivalent to a hinge when human foot contacts the ground. The external load " $F_{l}$ " including the exoskeleton mass and effective load hung at the back frame. The force " $F_{p}$ " provided by hydraulic actuator compensates the load on the exoskeleton.

The force and moment equilibrium equations of $1^{\#}$ linkage (synthetic calf) are shown as

$$
\begin{aligned}
& F_{\mathrm{O} 1 x}-F_{\mathrm{O} 2 x}+F_{\mathrm{O} 3 x}=m_{s c} a_{\mathrm{Gsx}}, \\
& F_{\mathrm{O} 1 y}+F_{\mathrm{O} 2 y}-F_{\mathrm{O} 3 y}-m_{s c} g=m_{s} a_{\mathrm{Gsy}}, \\
& T_{\mathrm{O} 1}+F_{\mathrm{O} 2 x} l_{s c} \cos \theta_{1}+F_{\mathrm{O} 2 y} l_{s c} \sin \theta_{1} \\
& \quad-F_{\mathrm{O} 3 x}\left(l_{s c}-l_{1}\right) \cos \theta_{1}-F_{\mathrm{O} 3 y}\left(l_{s c}-l_{1}\right) \sin \theta_{1} \\
& \quad-m_{s c} g l_{g s} \sin \theta_{1}=-I_{s c} \alpha_{\mathrm{O} 1},
\end{aligned}
$$

where $a_{n s}=l_{g s} \cdot \omega_{\mathrm{O} 1}^{2}, a_{\tau s}=l_{g s} \cdot \alpha_{\mathrm{O} 1}, a_{\mathrm{O} 1 x}=a_{\mathrm{O} 1 y}=0$.

Similarly, the $2^{\#}$ linkage (synthetic thigh), $3^{\#}$ linkage (piston), and $4^{\#}$ linkage (cylinder) can also be analyzed as follows:

$$
\begin{aligned}
& F_{\mathrm{O} 2 x}+F_{\mathrm{O} 4 x}=m_{t} a_{\mathrm{G} t x}, \\
& F_{\mathrm{O} 4 y}-F_{\mathrm{O} 2 y}-m_{t c} g-F_{\text {load }}=m_{t} a_{\mathrm{G} t y}, \\
& -F_{l} l_{t c} \cos \left(\theta_{k}+\theta_{1}\right)+F_{\mathrm{O} 4 x} l_{2} \cos \left(\theta_{k}+\theta_{1}\right) \\
& \quad-F_{\mathrm{O} 4 y} l_{2} \sin \left(\theta_{k}+\theta_{1}\right)+m_{t} g l_{g t} \sin \theta_{k}=I_{t c} \alpha_{\mathrm{O} 2}, \\
& F_{p} \sin \theta_{2}-F_{A} \cos \theta_{2}+F_{\mathrm{O} 3 x}=m_{p} a_{\mathrm{G} p x},
\end{aligned}
$$




$$
\begin{aligned}
& -F_{p} \cos \theta_{2}-F_{A} \sin \theta_{2}+F_{\mathrm{O} 3 y}=m_{p} a_{G p y}, \\
& -F_{A} l_{r}+T_{A}=I_{p} \alpha_{p}, \\
& -F_{p} \sin \theta_{2}+F_{A} \cos \theta_{2}-F_{\mathrm{O} 4 x}=m_{c} a_{G c x}, \\
& F_{p} \cos \theta_{2}+F_{A} \sin \theta_{2}-F_{\mathrm{O} 4 y}=m_{c} a_{G c y}, \\
& -F_{A}\left(l_{h}-l_{r}\right)-T_{A}=I_{c} \alpha_{c} .
\end{aligned}
$$

Some angle and angular velocity relations in Figure 2 are as follows:

$$
\begin{aligned}
& \sin \theta_{1}=\frac{l_{2} \sin \theta_{k}}{\sqrt{l_{1}^{2}+l_{2}^{2}-2 l_{1} l_{2} \cos \theta_{k}}}, \\
& \sin \theta_{3}=\frac{\left(l_{2}-l_{t c}\right) \sin \theta_{k}}{\sqrt{l_{s c}^{2}+\left(l_{2}-l_{t c}\right)^{2}-2 l_{s c}\left(l_{2}-l_{t c}\right) \cos \theta_{k}}}, \\
& \theta_{2}=\theta_{3}-\theta_{1}, \\
& \omega_{p}=\omega_{O 1} \\
& \quad-\frac{l_{2} \cos \theta_{k} \omega_{k}+l_{2} \sin \theta_{k}\left(l_{1} l_{2} \sin \theta_{k} \omega_{k} / \sqrt{l_{1}^{2}+l_{2}^{2}-2 l_{1} l_{2} \cos \theta_{k}}\right)}{\cos \theta_{3}\left(l_{1}^{2}+l_{2}^{2}-2 l_{1} l_{2} \cos \theta_{k}\right)},
\end{aligned}
$$

$$
\omega_{c}=\omega_{\mathrm{O} 2}
$$

$$
-\frac{l_{1} \cos \theta_{k} \omega_{k}+l_{1} \sin \theta_{k}\left(l_{1} l_{2} \sin \theta_{k} \omega_{k} / \sqrt{l_{1}^{2}+l_{2}^{2}-2 l_{1} l_{2} \cos \theta_{k}}\right)}{\cos \left(\pi-\theta_{k}-\theta_{3}\right)\left(l_{1}^{2}+l_{2}^{2}-2 l_{1} l_{2} \cos \theta_{k}\right)},
$$

$\omega_{\mathrm{O} 2}=\omega_{k}+\omega_{\mathrm{O} 1}$.

In above equations, some parameters are defined as follows.

Description of Some Motion and Geometry Parameter. Consider the following:

$F_{\mathrm{O} 1 x}, F_{\mathrm{O} 1 y}$ : force from hinge $O_{1}$ to linkage 1,

$F_{\mathrm{O} 2 x}, F_{\mathrm{O} 2 y}$ : force from linkage 1 to linkage 2,

$F_{\mathrm{O} 3 x}, F_{\mathrm{O} 3 y}$ : force from linkage 1 to linkage 3,

$F_{\mathrm{O} 4 x}, F_{\mathrm{O} 4 y}$ : force from linkage 2 to linkage 4 ,

$a_{G s x}, a_{G s y}$ : calf acceleration at the center of gravity,

$a_{G t x}, a_{G t y}$ : thigh acceleration at the center of gravity,

$a_{\tau s}, a_{n s}$ : tangential and normal acceleration of calf acceleration,

$a_{\tau t}, a_{n t}$ : tangential and normal acceleration of thigh, $a_{\tau p}, a_{n p}$ : tangential and normal acceleration of piston, $a_{\tau c}, a_{n c}$ : tangential and normal acceleration of cylinder,

$l_{g s}$ : length between the center of gravity calf and the ankle,

$l_{g t}$ : length between the center of gravity thigh and the knee,

$l_{1}, l_{2}$ : distance between hydraulic cylinder calf, thigh connections, and knee,
$\omega_{\mathrm{O} 1}, \alpha_{\mathrm{O} 1}$ : angular velocity and angular acceleration of calf around the ankle,

$\omega_{\mathrm{O} 2}, \alpha_{\mathrm{O} 2}$ : angular velocity and angular acceleration of thigh around the knee,

$\omega_{k}$ : knee angular velocity,

$I_{p}$ : moment of inertia of piston around the leg connection point,

$T_{\mathrm{O} 1}$ : torque from point $O_{1}$,

$\omega_{p}, \alpha_{p}$ : angular velocity and angular acceleration of piston,

$\omega_{c}, \alpha_{c}$ : angular velocity and angular acceleration of cylinder,

$F_{p}$ : radial force along the piston,

$F_{A}$ : normal force perpendicular to cylinder,

$T_{A}$ : torque from point $\mathrm{O}_{3}$,

$\theta_{1}$ : angle between linkage 1 and vertical line,

$\theta_{2}$ : angle between piston and vertical line,

$\theta_{3}$ : angle between piston and linkage 1 ,

$\theta_{k}$ : knee angle,

$m_{p}, m_{c}:$ piston and cylinder mass,

$l_{h}$ : total length of hydraulic actuator,

$l_{r}$ : length of piston.

If knee angle $\theta_{k}$ and angular velocity $\omega_{k}$ are given, (1a)$(8 \mathrm{c})$ can get the analytical solution " $F_{p}$ " So load pressure " $P_{l}$ " of the hydraulic actuator can be computed by

$$
P_{l}=\frac{F_{p}}{\pi r_{p}^{2}}
$$

where " $r_{p}$ " is hydraulic cylinder diameter.

Now the person can realize 2 typical actions: walk and squat after wearing the exoskeleton. The motional range of knee angle is from $85^{\circ}$ to $155^{\circ}$ in the gait duration of these actions. In addition, the maximum external load on the back frame of exoskeleton is $60 \mathrm{~kg}$. Substituting some known parameters into (3a)-(9), the load pressure $P_{l}$ can be computed digital simulation method shown in Figure 3. So if the external Load " $F_{l}$ " does not exceed $90 \mathrm{~kg}$, the load pressure $P_{l}$ will not be more than $8 \mathrm{MPa}$. It means that the hydraulic supply pressure " $P_{s}$ " can be set as $8 \mathrm{MPa}$ to appropriately compensate the external load $60 \mathrm{~kg}$. If the person wears the exoskeleton with no effective load, the supply pressure is chosen as $4 \mathrm{MPa}$ to balance the exoskeleton mass.

2.3. Hydraulic Model. The electrohydraulic servo control system is also fixed on the exoskeleton. It has 2 different characteristics: the asymmetric hydraulic cylinder and the single-rod actuator. The dynamics of servo valve can be described as follows:

$$
\frac{x_{\mathrm{v}}}{u}=\frac{K_{\mathrm{sv}} \omega_{\mathrm{sv}}^{2}}{s^{2}+2 \zeta_{\mathrm{sv}} \omega_{\mathrm{sv}} s+\omega_{\mathrm{sv}}^{2}},
$$




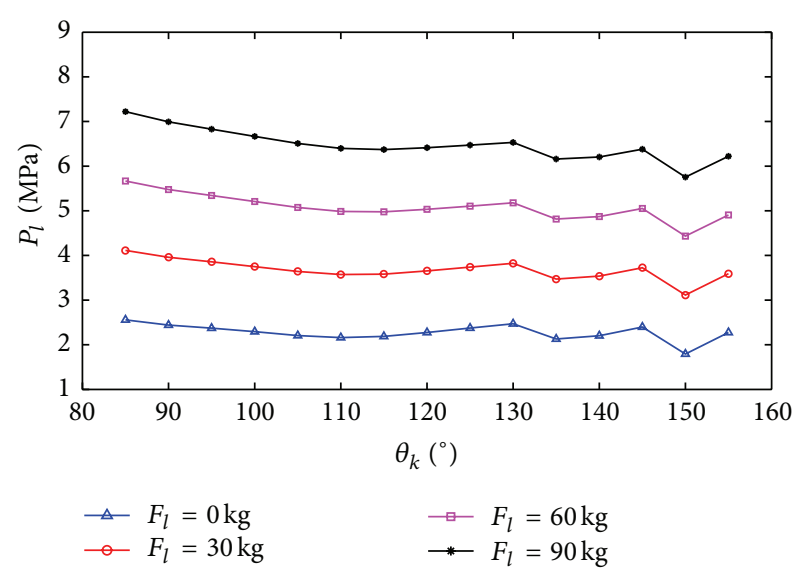

Figure 3: The load pressure $P_{l}$ in the gait duration of walk and squat varying load and knee angle.

where $\zeta_{\mathrm{sv}}$ is the damping ratio, $\omega_{\mathrm{sv}}$ is natural frequency, and $K_{\text {sv }}$ is the servo valve gain.

The flow equations can be linearized as follows [25]:

$$
\begin{aligned}
Q_{a} & =K_{q}^{1} x_{\mathrm{v}}-K_{c}^{1} p_{a}, \\
Q_{b} & =K_{q}^{2} x_{\mathrm{v}}+K_{c}^{2} p_{b}, \\
K_{q}^{1} & =C_{d} w \sqrt{\frac{2 \Delta p_{p}}{\rho},} \\
K_{q}^{2} & =C_{d} w \sqrt{\frac{2 \Delta p_{R}}{\rho},} \\
K_{c}^{1} & =\mid \frac{C_{d} w x_{\mathrm{v}}}{\sqrt{2 \rho \Delta p_{p}} \mid,} \\
K_{c}^{2} & =\mid \frac{C_{d} w x_{\mathrm{v}}}{\sqrt{2 \rho \Delta p_{R}} \mid,}, \\
\Delta p_{p} & = \begin{cases}\left|p_{s}-p_{a}\right| & x_{\mathrm{v}} \geq 0 \\
\left|p_{a}-p_{r}\right| & x_{\mathrm{v}}<0,\end{cases} \\
\Delta p_{R} & = \begin{cases}\left|p_{b}-p_{r}\right| & x_{\mathrm{v}} \geq 0 \\
\left|p_{s}-p_{b}\right| & x_{\mathrm{v}}<0,\end{cases}
\end{aligned}
$$

where $Q_{a}, Q_{b}$ are 2 sides of cylinder flow, $p_{a}, p_{b}$ are the cylinder chamber pressures, $p_{s}, p_{r}$ are supply and return pressures, $C_{d}$ is the valve coefficient of discharge, $w$ is the valve orifice area gradient, $\rho$ is the oil density, and $x_{\mathrm{v}}$ is the spool valve position. $K_{q}^{1}\left(K_{q}^{2}\right)$ and $K_{c}^{1}\left(K_{c}^{2}\right)$ are flow gains of the servo valve and flow-pressure coefficient.
If the internal and external leakages are neglected, the flow-pressure continuous equation of cylinder is shown as follows:

$$
\begin{aligned}
\dot{p}_{a} & =\frac{\beta_{e}}{V_{01}+A_{a} y}\left(Q_{a}-A_{a} \dot{y}\right), \\
\dot{p}_{b} & =\frac{\beta_{e}}{V_{02}-A_{b} y}\left(-Q_{b}+A_{b} \dot{y}\right), \\
V_{01} & =V_{02}=\frac{V_{t}}{2}=\frac{A_{a} l_{h}}{2},
\end{aligned}
$$

where $\beta_{e}$ is the fluid bulk modulus, $A_{a}$ and $A_{b}$ are the annulus areas of 2 sides of the cylinder, $V_{01}, V_{02}$ are half-volume of the cylinder, and $y$ is the cylinder position.

The mechanical dynamic equation is shown as follows:

$$
m \ddot{y}=\left(p_{a} A_{a}-p_{b} A_{b}-F_{f}-F_{p}\right),
$$

where $F_{f}$ is the stick-slip friction caused by the viscous damp of oil and $F_{p}$ is the variable load on the hydraulic actuator.

If the knee angle is known, the linear position of cylinder $y$ can be correspondingly computed by geometric relationship as follows:

$$
y\left(\theta_{k}\right)=\sqrt{l_{1}^{2}+l_{2}^{2}-2 \times l_{1} \times l_{2} \times \cos \theta_{k}} .
$$

According to the analytical computation method of the load pressure $P_{l}$ by (9), the linear expression between $F_{p}$ and $y$ can be constructed as follows:

$$
F_{p}\left(\theta_{k}, F_{L}\right)=K_{F_{p}}\left(\theta_{k}\right) y\left(\theta_{k}\right),
$$

where $K_{F_{p}}$ represents a variable bounded scale with load and knee angle.

The stick-slip friction often includes 2 items: a stick phase $F_{f \text { static }}$ which occurs when velocity is within a small critical velocity range and a slip friction $F_{f \text { slip }}$ which is the same as Coulomb friction model [26]. Here the slip friction can be estimated by simplified parameter identification method. Here the stick item is neglected, so the stick-slip friction $F_{f}$ can be approximately described as follows:

$$
F_{f} \approx b \dot{y},
$$

where $b$ is the viscous damping coefficient of oil.

The slip friction $F_{f s l i p}$ should be constructed in the simulation model to identify the parameters $b$. The maximum stroke of cylinder is controlled by one best PID control parameters to track the square demand. The comparison results are shown in Figure 4. The simulation result of hydraulic position is similar to the experiment when parameters $b$ are 100 , especially the dynamic response is better than other conditions. So the nominal value of $b$ can be approximately identified to be used in the parametric uncertainty model in Section 3.

\section{Robust Controller Design}

Lower extremity exoskeleton provides two features: high load bearing and fast motion. So exoskeleton should have fast 


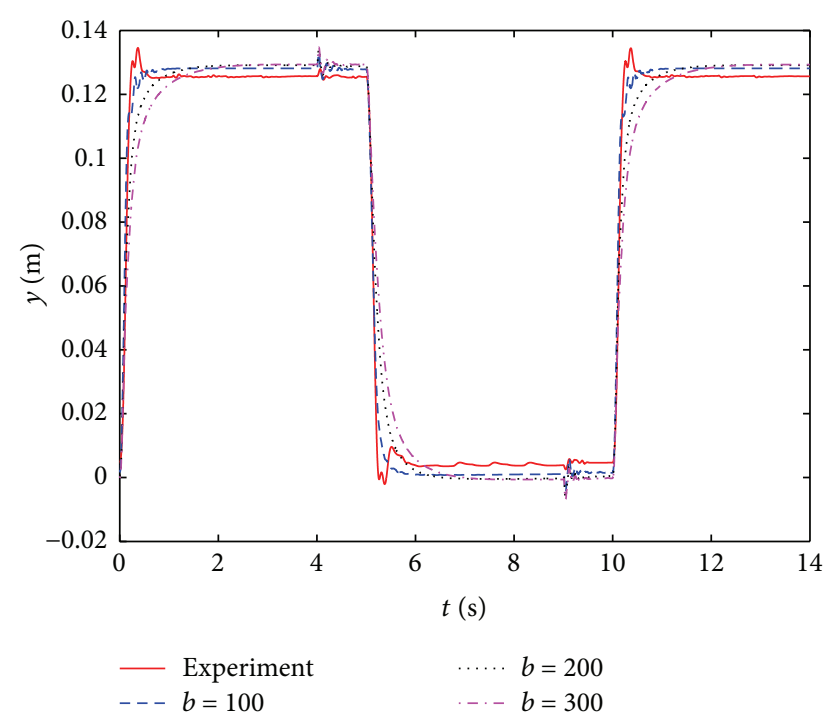

Figure 4: The square response of experiment and simulation varying the viscous damping coefficient $b$.

responses but should not hinder the wearer. In this section, a robust controller is designed to improve the dynamic response. The knee angle will be servo controlled to realize the human-machine coordinated motion. Because this EHSS has parametric disturbances and structural uncertainties, the robust $H_{\infty}$ control method is presented to satisfy the requirement of close-loop stability and robust performance. After the uncertainty model of the EHSS is constructed, the robust controller is designed to find the $H_{\infty}$ suboptimal controller.

3.1. Uncertainty Model Construction. This EHSS has some parametric uncertainties: the flow gains of servo valve $K_{q}^{i}$ $(i=1,2)$, the viscous damping coefficient $b$, the effective bulk modulus $\beta$ and variable volume of 2 sides cylinder $\left(V_{01}+A_{a} y, V_{02}-A_{b} y\right)$, and a structural uncertainty $K_{F_{p}}$ from external load. These uncertainties parameters $K_{q}, E, b$, and $K_{F_{p}}$ are described by upper Linear Fractional Transformation $[27,28]$ as follows:

$$
\begin{aligned}
M_{K_{q}} & =\left[\begin{array}{cc}
0 & \bar{K}_{q} \\
p_{K_{q}} & \bar{K}_{q}
\end{array}\right], \\
M_{E} & =\left[\begin{array}{cc}
-p_{E} & \overline{\bar{E}} \\
-p_{E} & \overline{\bar{E}}
\end{array}\right], \\
M_{b} & =\left[\begin{array}{cc}
0 & \bar{b} \\
p_{b} & \bar{b}
\end{array}\right], \\
M_{K_{F p}} & =\left[\begin{array}{cc}
0 & 1 \\
p_{K_{F p}} & \bar{K}_{F_{p}}
\end{array}\right],
\end{aligned}
$$

$$
\begin{aligned}
K_{q} & =\bar{K}_{q}\left(1+p_{K_{q}} \delta_{K_{q}}\right), \quad-1 \leq \delta_{K_{q}} \leq 1, \\
E & =\bar{E}\left(1+p_{E} \delta_{E}\right), \quad-1 \leq \delta_{E} \leq 1, \\
b & =\bar{b}\left(1+p_{b} \delta_{b}\right), \quad-1 \leq \delta_{b} \leq 1, \\
K_{F_{p}} & =\bar{K}_{F_{p}}+p_{K_{F p}} \delta_{K_{F p}},
\end{aligned}
$$

where $E=V_{t} / 2 \beta, \overline{K_{q}}, \bar{E}$, and $\bar{b}$ are the nominal values, $\delta_{K_{q}}, \delta_{E}$, and $\delta_{b}$ are relative variation, respectively, and the maximum relative uncertainties $p_{K_{q}}, p_{E}$, and $p_{b}$ can be estimated varying some hydraulic parameters [20].

If these vectors are defined, the state vector $X=$ $\left[x_{1}, x_{2}, x_{3}, x_{4}, x_{5}, x_{6}\right]^{T}=\left[x_{\mathrm{v}}, \dot{x}_{\mathrm{v}}, p_{a}, p_{b}, y, \dot{y}\right]^{T}$, the control input $u(t)$, which is the current of servo valve, the vector of exogenous inputs $w(t)=\left[w_{1}, w_{2}, w_{3}, w_{4}, w_{5}\right]^{T}$, the output cylinder position $y(t)$ measured by encoder, and the regulated output vector $z(t)=\left[z_{1}, z_{2}, z_{3}, z_{4}, z_{5}, z_{6}\right]^{T}$, then the uncertain state-space model is constructed as follows:

$$
\begin{aligned}
& \dot{X}(t)=A X(t)+B_{1} W(t)+B_{2} u(t), \\
& Z(t)=C_{1} X(t)+D_{11} W(t), \\
& Y(t)=C_{2} X(t)
\end{aligned}
$$

where

$$
\begin{aligned}
& A=\left[\begin{array}{cccccc}
0 & 1 & 0 & 0 & 0 & 0 \\
-\omega_{\mathrm{v}}^{2} & -2 \zeta_{\mathrm{v}} \omega_{\mathrm{v}} & 0 & 0 & 0 & 0 \\
\bar{K}_{q} & 0 & -\frac{K_{c}}{\bar{E}} & 0 & 0 & -\frac{A_{a}}{\bar{E}} \\
\frac{\bar{E}}{\bar{K}_{q}} & 0 & 0 & -\frac{K_{c}}{\bar{E}} & 0 & \frac{A_{2}}{\bar{E}} \\
-\frac{\bar{E}}{\bar{E}} & 0 & 0 & 0 & 0 & 1 \\
0 & 0 & \frac{A_{a}}{m} & -\frac{A_{b}}{m} & -\frac{\bar{K}_{F_{p}}}{m} & \frac{\bar{b}}{m}
\end{array}\right] \text {, } \\
& B_{1}=\left[\begin{array}{ccccc}
0 & 0 & 0 & 0 & 0 \\
0 & 0 & 0 & 0 & 0 \\
\frac{p_{K_{q}}}{\bar{E}} & -p_{E} & 0 & 0 & 0 \\
-\frac{p_{K_{q}}}{\bar{E}} & 0 & -p_{E} & 0 & 0 \\
0 & 0 & 0 & 0 & 0 \\
0 & 0 & 0 & -\frac{p_{b}}{m} & -\frac{p_{K_{F p}}}{m}
\end{array}\right] \text {, }
\end{aligned}
$$




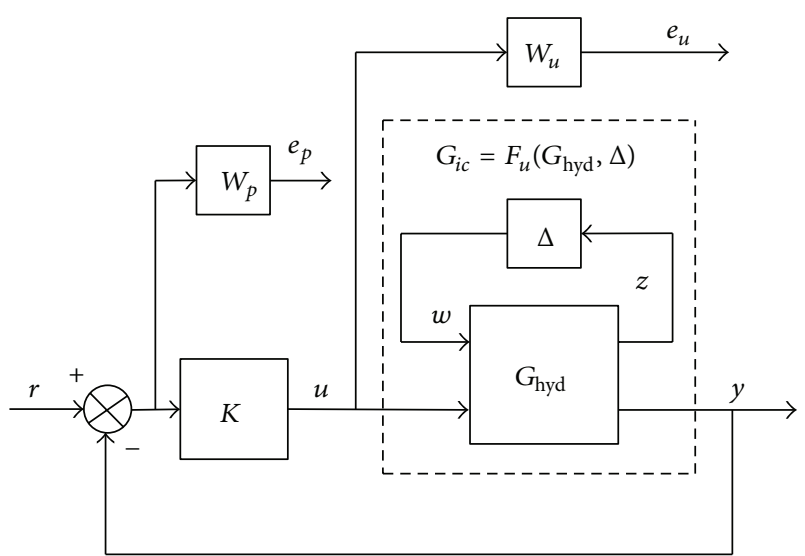

FIGURE 5: The block diagram of the closed-loop system with robust stability and performance requirements.

$$
\begin{aligned}
B_{2}= & {\left[\begin{array}{llllll}
0 & K_{\mathrm{sv}} \omega_{\mathrm{v}}^{2} & 0 & 0 & 0 & 0
\end{array}\right]^{T}, } \\
C_{1} & =\left[\begin{array}{cccccc}
\bar{K}_{q} & 0 & 0 & 0 & 0 & 0 \\
\frac{K_{q}}{\bar{E}} & 0 & -\frac{K_{c}}{\bar{E}} & 0 & 0 & -\frac{A_{a}}{\bar{E}} \\
\frac{\bar{K}_{q}}{\bar{E}} & 0 & 0 & -\frac{K_{c}}{\bar{E}} & 0 & \frac{A_{b}}{\bar{E}} \\
0 & 0 & 0 & 0 & 0 & \frac{\bar{b}}{b} \\
0 & 0 & 0 & 0 & 1 & 0
\end{array}\right], \\
D_{11} & =\left[\begin{array}{cccccc}
0 & 0 & 0 & 0 & 0 \\
\frac{p_{K_{q}}}{\bar{E}} & -p_{E} & 0 & 0 & 0 \\
-\frac{p_{K_{q}}}{\bar{E}} & 0 & -p_{E} & 0 & 0 \\
0 & 0 & 0 & 0 & 0 \\
0 & 0 & 0 & 0 & 0
\end{array}\right], \\
C_{2}= & {\left[\begin{array}{cccccc}
0 & 0 & 1 & 0
\end{array}\right] . }
\end{aligned}
$$

The robust stability and performance requirements of the closed-loop system are shown in Figure 5.

In Figure 5, the weighting functions $W_{p}$ and $W_{u}$ are used to reflect the relative significance of the performance requirement. The robust controller $K$ needs to be designed to optimize the robust performance $e_{p}$ and $e_{u}$. The nominal model $G_{\text {hyd }}$ and the interconnection model $G_{\text {ic }}$ represent the robust uncertainty model shown in (22a), (22b), and (22c). The demand and actual cylinder position $r, y$ are the input and output of this EHSS.

3.2. Robust Controller Design. The frequency response of the open-loop system with model uncertainties is shown in Figure 6 . The phase margins are obviously different between perturbed model and nominal model, which is caused by the uncertainty parameter $K_{F_{p}}$. Due to the presence of $K_{F_{p}}$, the stable phase of open-loop system will lead or lag
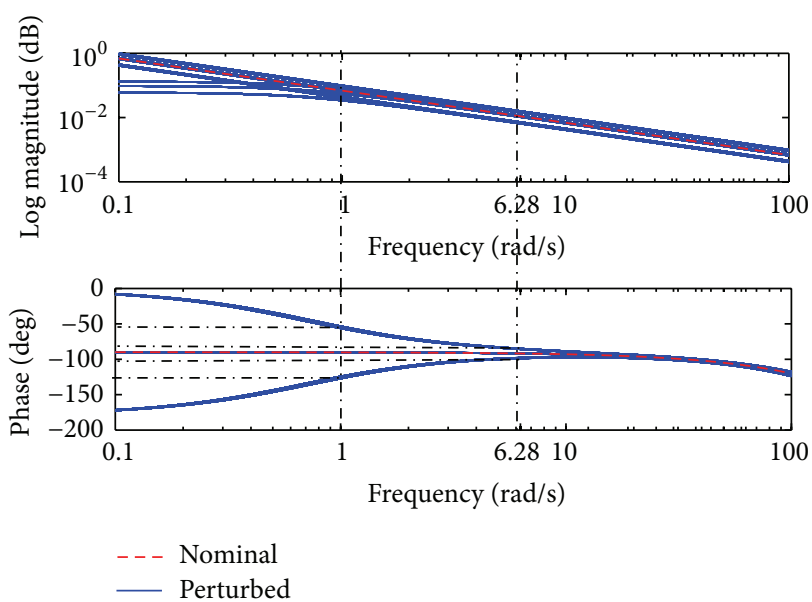

FIGURE 6: The frequency response of the open-loop system with varying uncertainty parameters $K_{q}, E, b$, and $K_{F_{p}}$.

approximately 50 degrees in middle frequency stage (1 $6.28 \mathrm{rad} / \mathrm{s}$ ).

The weight functions $W_{p}$ and $W_{u}$ are iteratively designed according to robust performance requirement and control constraint shown as follows [29]:

$$
\begin{aligned}
& W_{p}=0.95 \times \frac{s^{2}+1.8 s+5}{s^{2}+8 s+0.01}, \\
& W_{u}=5 \times 10^{-4} .
\end{aligned}
$$

The singular values of the weight function $W_{p}$ and the sensitivity function of close-loop $G_{s}^{n}$ should satisfy $\left\|W_{p} G_{s}^{n}\right\|_{\infty} \leq \mu\left(W_{p}\right) \mu\left(G_{s}^{n}\right)<1$, which has guaranteed the basic control performance.

The robust controller will guarantee robust stability and robust performance requirement which can be described in Figure $5[30,31]$.

(1) $H_{\infty}$ suboptimal control problem: to design a controller $K$ satisfying the following condition:

$$
\left\|F_{l}\left(G_{\mathrm{ic}}, K\right)\right\|_{\infty}=\left\|F_{l}\left(F_{u}\left(G_{\text {hyd }}, \Delta\right), K\right)\right\|_{\infty}<\gamma,
$$

where $\gamma>\gamma_{0}, \gamma_{0}$ is the minimum value of $\left\|F_{l}\left(G_{\mathrm{ic}}, K\right)\right\|_{\infty}$ under $K$ is a stable controller.

(2) Robust performance requirement: the close-loop system composed of $G_{\text {ic }}$ and $K$ should satisfy

$$
\left\|\left[\begin{array}{c}
W_{p}\left(I+G_{\mathrm{ic}} K\right)^{-1} \\
W_{u} K\left(I+G_{\mathrm{ic}} K\right)^{-1}
\end{array}\right]\right\|_{\infty}<1 .
$$

If the relative uncertainty $\Delta$ is from $-I$ to $\Delta=I$, the singular value of close-loop perturbed is 0.28 , which is less than 1 shown in Figure 7. The close-loop system $G_{c}$ is stable and its relative uncertainties $\Delta$ can be extended to $1 / 0.28$.

In Figure 8 , the maximum singular value $\mu$ of close-loop shows that the robust performance requirement is 1.4 , which 


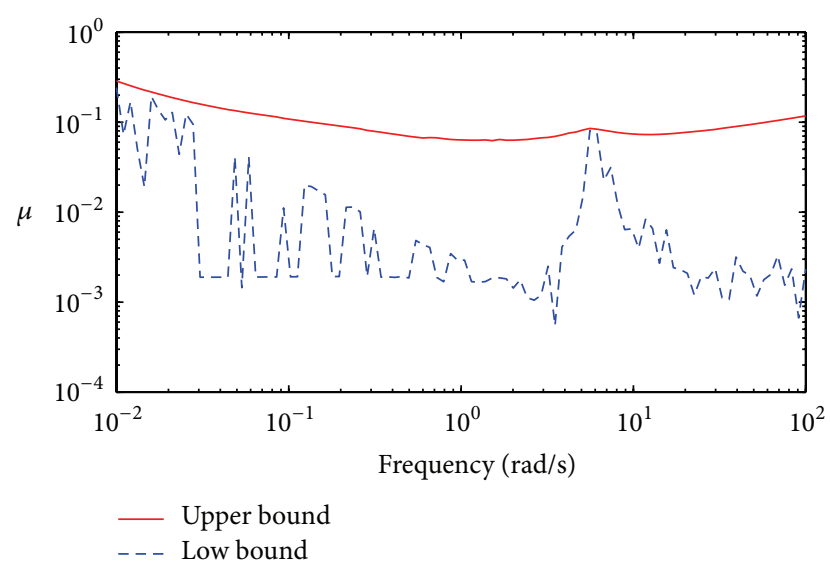

FIGURE 7: The maximum robust stability bound with varying uncertainty parameters $K_{q}, E, b$, and $K_{F_{p}}$.

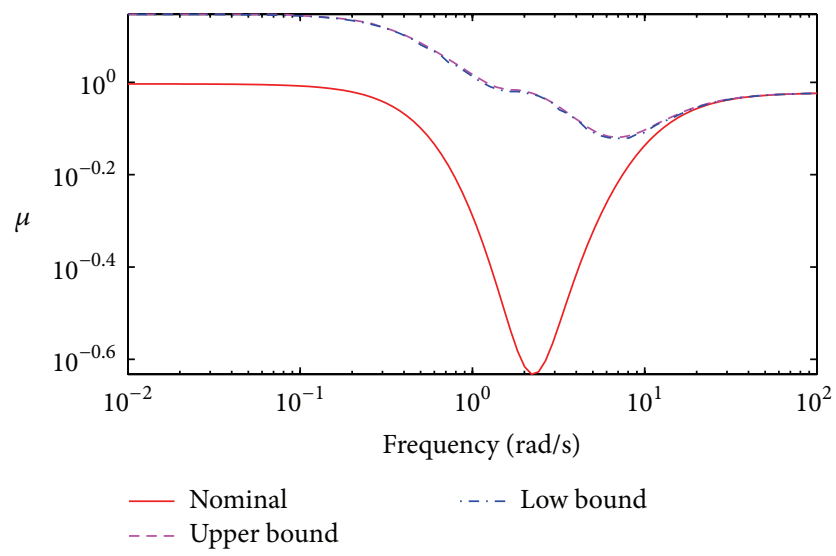

FIGURE 8: The maximum robust performance bound with varying uncertainty parameters $K_{q}, E, b$, and $K_{F_{p}}$.

means that the steady tracking accuracy may not be excellent. But in middle and high frequency stage from 4 to $100 \mathrm{rad} / \mathrm{s}$, the dynamic tracking performance and robustness margin meet the requirement [32].

After iterative design, the robust controller is 7 orders. Taking the actual project into account, the controller should be reduced into lower order by Balanced Truncation Method [33]. So the controller is simplified to 4 orders as follows:

$$
\begin{aligned}
u_{\text {extension }} & =K(s)(\tilde{y}-y) \\
& =\frac{n_{4} s^{4}+n_{3} s^{3}+n_{2} s^{2}+n_{1} s+n_{0}}{d_{4} s^{4}+d_{3} s^{3}+d_{2} s^{2}+d_{1} s+d_{0}}(\tilde{y}-y),
\end{aligned}
$$

where $n_{i}(i=0, \ldots, 4), d_{i}(i=0, \ldots, 4)$ are numerator and denominator constant, $\tilde{y}$ is demand input, and $y$ is output.

\section{Gait Divisions of Coordinated Motion}

In this section, the gait phase divisions of 2 typical action modes are analyzed and designed. According to the gait identification, the switched control is realized to reduce

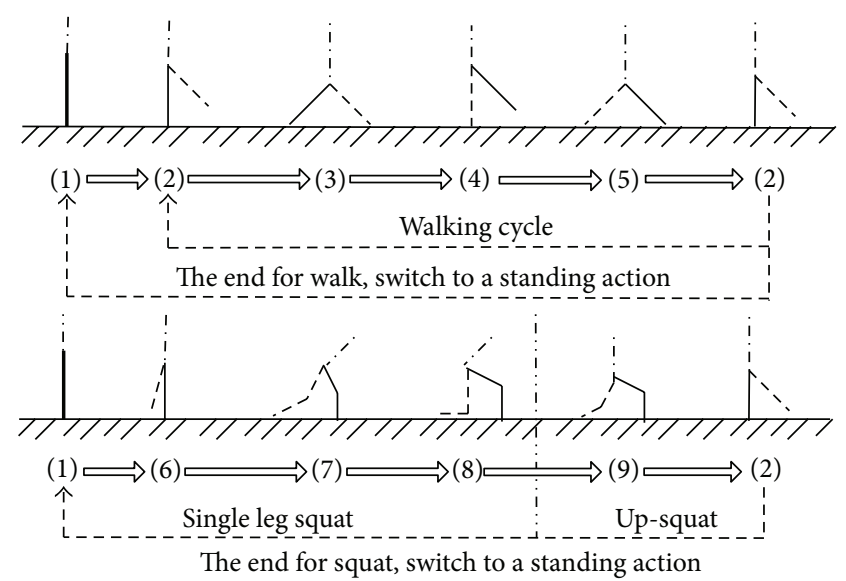

-.-. Upper limb trunk _ - - Right leg (thigh and shank)

_ Left leg (thigh and shank)

FIGURE 9: The gait subphases division of walk and squat action in human-machine coordinated motion.

the delay of exoskeleton tracking person and improve the human-machine coupling effect.

4.1. Walk Mode. The walking gait law of human-machine coordinate motion can be divided into 5 subphases shown in Figure 9. These subphases are (1) double standing; (2) right leg swing, left leg stance; (3) double stance with right leg front and left leg back; (4) right leg stance and left leg swing; and (5) double stance with left leg front and right leg back.

In order to improve the exoskeleton tracking person's motion, 2 subphases (3) and (4) are merged. That means if the subphases (3) are identified, make the hydraulic cylinder of left leg retract in advance. This operation realizes the exoskeleton ahead of person, which helps person regulate the center of system gravity easily in overload carrying condition. After the subphase (4) is identified by exoskeleton, the center of system gravity is steadily regulated by person. Simultaneously, the exoskeleton continuously realizes the right stance leg and the left swing leg. The subphases (5) and (2) are similar to subphases (3) and (4). So the switched control algorithm is described in Table 1.

In Table $1, u_{l}$ and $u_{r}$ are control current of the of left and right servo valve, $u_{\max }= \pm 40 \mathrm{~mA}$, which is the maximum current of servo.

4.2. Squat Mode. The squat gait law is divided into 6 subphases: (1) double standing; (6) left leg stance and right leg uplift for preparation; (7) left leg retraction gradually and right leg swing; (8) left leg reached the minimum position of cylinder and right knee contacting the ground, which means the single leg squat action ends; (9) left leg extension gradually and right leg swing; and (2) left leg stance and right leg swing, which means the up-squat action ends and switch to the walking mode. So the switched control algorithm is described in Table 2.

In gait (7), the left knee angle is retracted by fader control. The symbol $k=2$ and $t$ is fader time. 
TABLE 1: The duty of exoskeleton and person in walking gait subphases by different switched controller form.

\begin{tabular}{|c|c|c|c|}
\hline Gait & Controller & Exoskeleton & Person \\
\hline (1) & $\begin{array}{l}u_{l}=u_{\text {extension }} \\
u_{r}=u_{\text {extension }}\end{array}$ & $\begin{array}{c}\text { Control double } \\
\text { knee angle }\end{array}$ & $\begin{array}{c}\text { Adapt to } \\
\text { exoskeleton }\end{array}$ \\
\hline (2) & $\begin{array}{c}u_{l}=u_{\text {extension }} \\
u_{r}=-u_{\max }\end{array}$ & $\begin{array}{l}\text { Control left } \\
\text { knee angle and } \\
\text { retract right leg }\end{array}$ & $\begin{array}{c}\text { Adapt to } \\
\text { exoskeleton }\end{array}$ \\
\hline (3) & $\begin{array}{c}u_{l}=-u_{\max } \\
u_{r}=u_{\text {extension }}\end{array}$ & $\begin{array}{l}\text { Adapt to person } \\
\text { and retract left } \\
\text { leg in advance }\end{array}$ & $\begin{array}{l}\text { Regulate the } \\
\text { center of gravity }\end{array}$ \\
\hline (4) & $\begin{array}{c}u_{l}=-u_{\max } \\
u_{r}=u_{\text {extension }}\end{array}$ & $\begin{array}{l}\text { Control right } \\
\text { knee angle and } \\
\text { retract left leg }\end{array}$ & $\begin{array}{c}\text { Adapt to } \\
\text { exoskeleton }\end{array}$ \\
\hline (5) & $\begin{array}{c}u_{l}=u_{\text {extension }} \\
u_{r}=-u_{\max }\end{array}$ & $\begin{array}{l}\text { Adapt to person } \\
\text { and retract right } \\
\text { leg in advance }\end{array}$ & $\begin{array}{l}\text { Regulate the } \\
\text { center of gravity }\end{array}$ \\
\hline
\end{tabular}

TABLE 2: The duty of exoskeleton and person in squat gait subphases by different switched controller form.

\begin{tabular}{cccc}
\hline Gait & Controller & Exoskeleton & Person \\
\hline (6) & $\begin{array}{c}u_{l}=u_{\text {extension }} \\
u_{r}=-u_{\max }\end{array}$ & $\begin{array}{c}\text { Control left } \\
\text { knee angle and } \\
\text { retract right leg }\end{array}$ & $\begin{array}{c}\text { Squat } \\
\text { preparation }\end{array}$ \\
\hline (7) & $\begin{array}{c}u_{l}= \\
u_{\max }\left(1-e^{-k t}\right) \\
u_{r}=-u_{\max }\end{array}$ & $\begin{array}{c}\text { Retract left knee } \\
\text { angle gradually }\end{array}$ & $\begin{array}{c}\text { Adapt to } \\
\text { exoskeleton }\end{array}$ \\
\hline (8) & $\begin{array}{l}\text { Adapt to person } \\
\text { and hold in the } \\
\text { prescript } \\
\text { posture }\end{array}$ & $\begin{array}{c}\text { Regulate the } \\
\text { center of gravity }\end{array}$ \\
\hline$u_{r}=-u_{\max }$ & $\begin{array}{c}\text { Control 2 knee } \\
\text { angles and } \\
\text { extend 2 legs }\end{array}$ & $\begin{array}{c}\text { Adapt to } \\
\text { exoskeleton }\end{array}$ \\
\hline
\end{tabular}

To ensure the safety of person, the squat preparation time need to be set as 2 or $3 \mathrm{sec}$. The subphase (8) is identified by a pressure force sensor on the right knee. The transition from subphase (8) to subphase (9) needs a mode switch button and gait identification algorithm which is not described in this section.

\section{Simulation Results}

The hydraulic model is simulated with the external load $60 \mathrm{~kg}$. The hydraulic parameters for simulation are shown in Table 3. In order to verify the effectiveness of the proposed method, the natural walking gait law is considered as the command input with $0.5 \mathrm{~Hz}$ frequency. The fore-half duration is stance phase for one leg with the range of knee angle from $15^{\circ}$ to $30^{\circ}$. The afterwards duration is swing phase with range of knee angle from $15^{\circ}$ to $73^{\circ}$.

According to the above analysis of the load pressure $P_{l}$, the hydraulic supply pressure $P_{s}$ is $8 \mathrm{MPa}$ and the external load is $60 \mathrm{~kg}$ in simulation. Some hydraulic parameters are shown in Table 3. The maximum relative uncertainties $p_{K_{q}}=0.25$,
TABLE 3: The hydraulic parameters for simulation.

\begin{tabular}{lcc}
\hline Name & Value & Unit \\
\hline Damping ratio $\xi_{\mathrm{sv}}$ & 0.7 & - \\
Natural frequency $\omega_{n}$ & 628 & $\mathrm{rad} / \mathrm{s}$ \\
Servo valve gain $K_{\mathrm{sv}}$ & 0.0125 & $\mathrm{~m} / \mathrm{A}$ \\
Fluid bulk modulus $\beta_{e}$ & 7000 & $\mathrm{bar}$ \\
Mass of hydraulic actuator $m$ & 2.5 & $\mathrm{~kg}$ \\
Annulus areas of no-rod cylinder $A_{a}$ & $1.77 \times 10^{-4}$ & $\mathrm{~m}^{2}$ \\
Annulus areas of rod cylinder $A_{b}$ & $1.13 \times 10^{-4}$ & $\mathrm{~m}^{2}$ \\
Distance between hydraulic cylinder & 0.322 & $\mathrm{~m}$ \\
calf connections and knee $l_{1}$ & & \\
Distance between hydraulic cylinder & 0.065 & $\mathrm{~m}$ \\
thigh connections and knee $l_{2}$ & 1 & $\mathrm{bar}$ \\
Return pressures $p_{r}$ & 0.16 & $\mathrm{~m}$ \\
Piston stroke $L_{r}$ & $5.18 e-12$ & $\mathrm{~m}^{3} / \mathrm{s} / \mathrm{Pa}$ \\
Flow-pressure coefficient $K_{c}^{1} \approx K_{c}^{2}$ & 0.188 & $\mathrm{~m} / \mathrm{s}$ \\
Nominal value of $\bar{K}_{q}$ & 100 & $\mathrm{Ns} / \mathrm{m}$ \\
Nominal value of $\bar{b}$ & $1.98 e-14$ & $\mathrm{~m} / \mathrm{pa}$ \\
Nominal value of $\bar{E}$ & 1200 & $\mathrm{~N} / \mathrm{m}$ \\
Nominal value of $\bar{K}_{F_{p}}$ & &
\end{tabular}

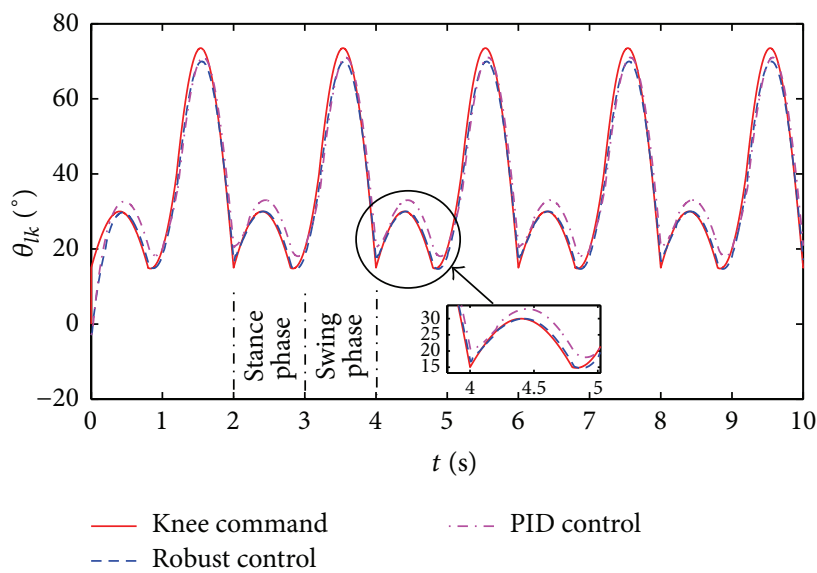

FIGURE 10: The left knee angle response of walking gait.

$p_{E}=0.7, p_{b}=1$, and $p_{K_{F p}}=300$. Here 2 control methods are used to make the exoskeleton track the person in the walking mode simulation. The PID control parameters are designed as the best group, which satisfy the frequency domain margin. The robust $H_{\infty}$ control method is realized by (27).

The left knee angle response of exoskeleton is shown in Figure 10. In swing phase, the dynamic characteristic of PID control is same as the robust $H_{\infty}$ control because there is no external load on the hydraulic actuator. But, in stance phase, the dynamic performance of robust control is better than PID control due to the variable compensation force $F_{p}$ provided by hydraulic actuator on the exoskeleton. This external force disturbance results in the parametric uncertainty of $K_{q}$, E, which reduce the stabile margin of PID control. Importantly, the control current needs to balance the compensation force $F_{p}$ and stick-slip friction $F_{f}$ in the case 


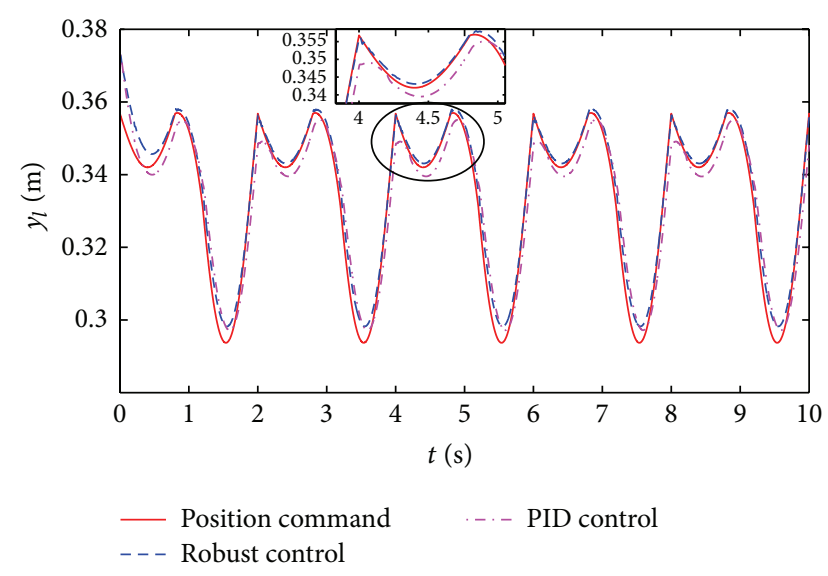

FIGURE 11: The position response of left hydraulic cylinder of walking gait.

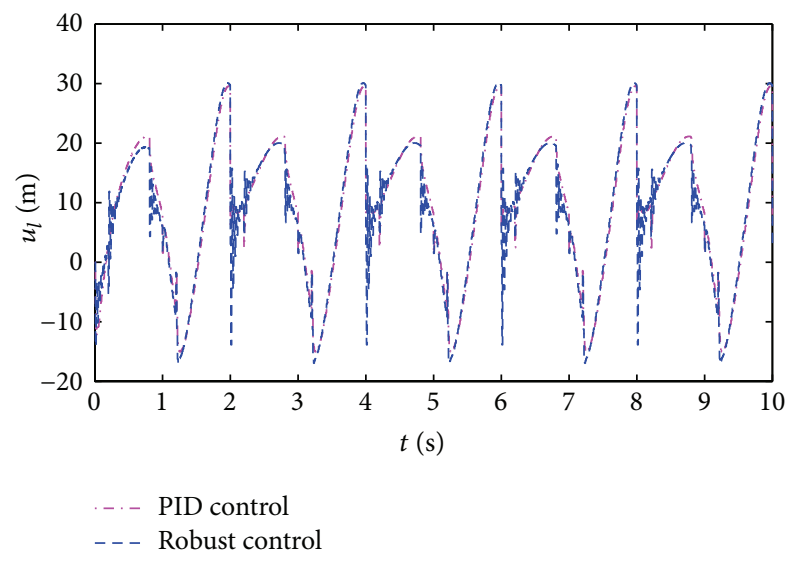

FIGURE 12: The left servo valve control current of walking gait.

of positional error suppression. In addition, the asymmetric cylinder affects the dynamic characteristic of hydraulic model when cylinder extension switches into retraction, especially in stance phase. The parametric uncertainty of $K_{q}, E$, and $b$ and structural uncertainty $F_{p}$ are considered in the system model construction, so the robust controller can guarantee the dynamic performance in the whole duration of walking mode.

The dynamic cylinder position of left hydraulic actuator on exoskeleton is shown in Figure 11. It is computed by (17) after the knee angle is measured by encoder. The available displacement of cylinder is $0.07 \mathrm{~m}$ in waking mode, which is less than the maximal stroke $0.16 \mathrm{~m}$ of cylinder. It means that only half stroke is useful in human-machine coordinated walk. But the maximal stroke of cylinder needs to satisfy the squat mode, which requires enough length of cylinder.

The control current of left servo valve is shown in Figure 12. The general trend of control current is same using 2 control methods. But the robust controller $u_{\text {extension }}$ is more intense than PID control, especially in stance phase. Because the controller involves the high order item, the robust controller can reduce the tracking error quickly in the

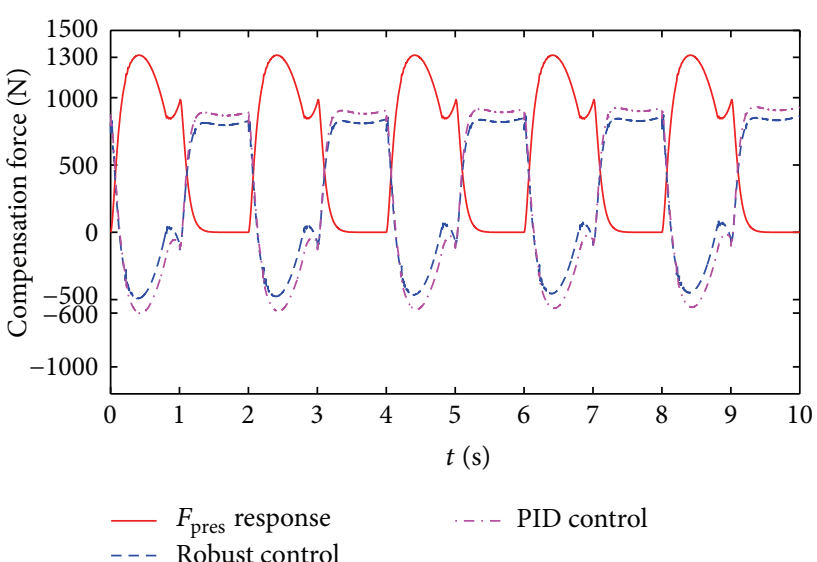

Figure 13: The aid-force effect of exoskeleton by left hydraulic actuator.

case of model uncertainty. The maximal amplitude of control current is $30 \mathrm{~mA}$, which does not exceed the maximal servo valve control current $\pm u_{\max }$.

The aid-force effect of exoskeleton by left hydraulic actuator is shown in Figure 13. Here the demand compensation force $F_{\text {pres }}$ is constructed by

$$
F_{\text {pres }}= \begin{cases}\frac{F_{p}}{\left(T_{\alpha} s+1\right)^{2}} & \text { stance phase } \\ \left(1-\frac{1}{\left(T_{\alpha} s+1\right)^{2}}\right) F_{p} & \text { swing phase }\end{cases}
$$

where $T_{\alpha}=0.05 \mathrm{~s}$ is defined as the transition response time of external load $F_{l}$.

In Figure 13, the compensation force error means the error between $F_{\text {pres }}$ and the hydraulic output actual force $F_{\text {act }}$. In stance phase of left leg, the compensation force error does not exceed $500 \mathrm{~N}$ by the robust controller. The demand compensation force $F_{\text {pres }}$ is $1300 \mathrm{~N}$ approximately. It means that the load bearing efficiency is great than $60 \%$ in stance phase of left leg. Because 2 legs are alternative in walking gait, the left hydraulic actuator needs not to compensate $F_{\text {pres }}$ in its swing phase, while the hydraulic actuator on right leg of exoskeleton is in charge of this load bearing task. Therefore, the aid-force effect of simulation result can not include the hydraulic output actual force in swing phase in this figure.

\section{Experimental Results}

The human-machine coordinated motion is realized by a tester wearing the exoskeleton prototype. The gait can be identified by 4 force sensors in the sensor shoes. The 2 knee angles of exoskeleton are measured by 2 encoders. The hydraulic control system includes a motor, a fixed pump, 2 servo valves, 2 cylinders, and an embedded controller on the mechanical brace. The net weight of prototype is $30 \mathrm{~kg}$. In walking mode, the tester can bear no more than $60 \mathrm{~kg}$ external load. In squat mode, the tester can realize the basic gait with no external load due to the safety of 


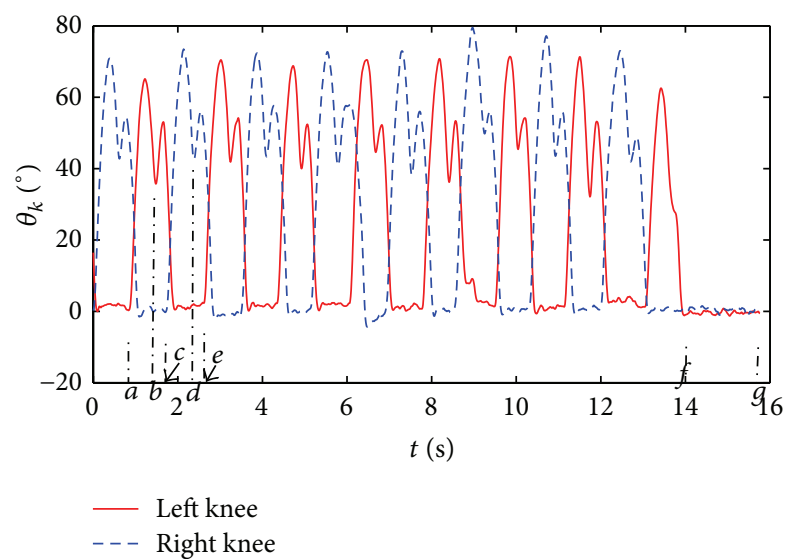

FIGURE 14: The knee angles of 2 legs in walking mode.

tester. The hydraulic actuators are controlled by the robust control method and the control algorithm will be switched in different gait.

6.1. Walking Mode with Overloading. In walking mode, the extension and retraction of 2 legs are alternatively realized by hydraulic actuator. The walking frequency is $0.5 \mathrm{~Hz}$ bearing with $60 \mathrm{~kg}$ load.

The dynamic characteristics of 2 knee angles are shown in Figure 14, which includes 5 subphases of walking gait. In gait (4), described by time slice $a \sim b(0.8 \mathrm{~s} \sim 1.5 \mathrm{~s})$, the left leg is retracted by the tester himself and the control current of left leg is $-u_{\max }$ shown in Figure 20. In gait (5), described by time slice $b \sim c(1.5 \mathrm{~s} \sim 1.7 \mathrm{~s})$, the left leg is extended by feedback control and the right leg begins to retract in advance to help the tester regulate the center of gravity. This ahead of control can make the tester move easily. In gait (2), described by time slice $c \sim d(1.7 \mathrm{~s} \sim 2.4 \mathrm{~s})$, the right leg is retracted by the tester himself like as gait (4). In gait (3), described by time slice $d \sim e$ (2.4 s 2.6 s), the right leg is extended by feedback control like gait (5). These 4 subphases are switched frequently in walking mode. If the walking mode ends, the double stance phase gait (1) will arise, described by time slice $f \sim g(14 s \sim 15.6 s)$. In gait (1), 2 legs are controlled by feedback control. The external load is compensated by 2 hydraulic actuators on the exoskeleton only in stance phase. In swing phase, the exoskeleton should help the tester switch the center of gravity from one leg to the other leg. Otherwise, the human-machine coupled effect is reduced significantly and the tester may be at loss of balance.

The cylinder positions of 2 hydraulic actuators in one cycle of walking mode are shown in Figure 15. Here the extended position commands are designed as a rising step. The servo feedback control phase of left leg is described as time slice $b \sim e(1.5 \mathrm{~s} \sim 2.6 \mathrm{~s})$. But, in time slice $b 1 \sim b 2(1.55 \mathrm{~s} \sim$ $1.65 \mathrm{~s})$, the cylinder position is still retracted after the positive control current output. This response delay is caused by the bandwidth limitation of the whole hydraulic control system. The pressure of cylinder is constructed with time-consuming $0.8 \mathrm{~s}$ from 0 to stable value $8 \mathrm{MPa}$. So the available position response of left cylinder is time slice $b 2 \sim e(1.65 \mathrm{~s} \sim 2.6 \mathrm{~s})$,

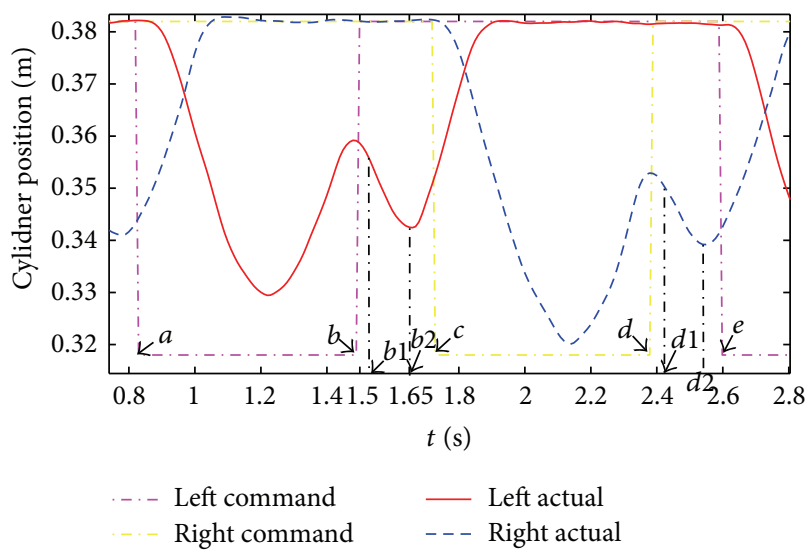

FIgURE 15: The cylinder positions of 2 hydraulic actuators in one cycle of walking mode.

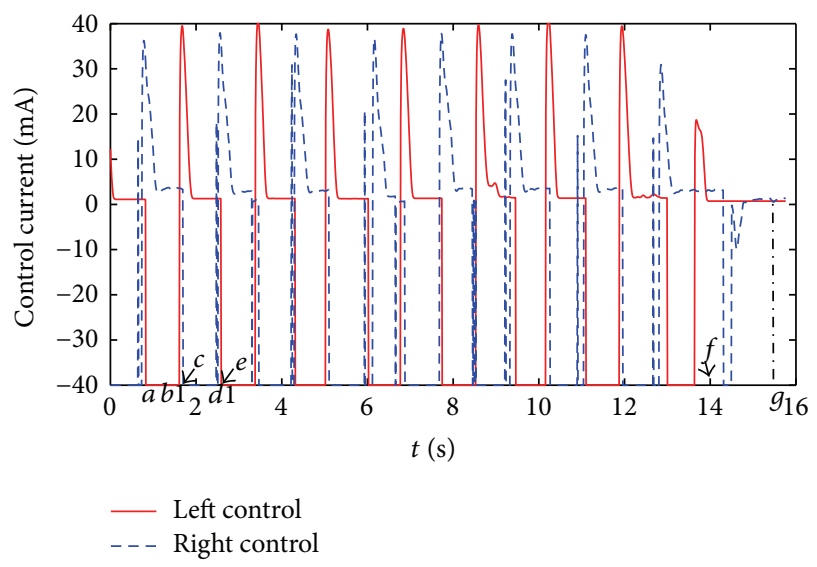

FIGURE 16: The control current of 2 servo valves in walking mode.

where the actual position of cylinder tracks the extended position commands. In time slice $a \sim b$, the retracted position commands of left leg is designed as a fall step. It is not a servo control phase for left leg. So the control current of servo valve is minus maximal value $-u_{\max }$ directly in order to help the tester retract the cylinder quickly. The dynamic characteristic of right cylinder positions is similar to the left cylinder.

The switched control currents correspond to 5 subphases shown in Figure 16. The servo control phase of left leg is time slice $b 1 \sim e(1.55 \mathrm{~s} \sim 2.6 \mathrm{~s})$ in one cycle. Similarly, the servo control phase of right leg is starting at time $d 1$. In double stance phase, described by time slice $f \sim g$, the servo control value is near 0 after 2 knee angles reach the tester's stance posture. The dynamic pressures of 2 hydraulic actuators are shown in Figure 17. In stance phase, the pressure is constructed from 0 to $8 \mathrm{MPa}$ with approximately $0.8 \mathrm{~s}$ time consumed due to bearing heavy load. In swing phase, the fast time-consuming is $0.4 \mathrm{~s}$ for pressure from $8 \mathrm{MPa}$ to 0 . It reveals that person's active control can be faster than the control response of exoskeleton if the walking speed is possible. So the switched control algorithm shown in Table 1 is suitable for human-machine coordinated walk. 


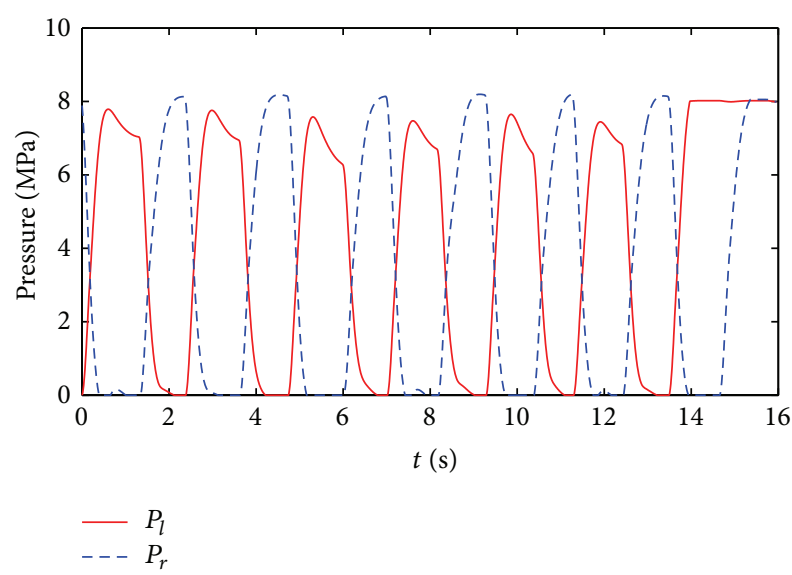

FIgURE 17: The pressures of 2 hydraulic actuators in walking mode.

6.2. Squat Mode Test. According to Figure 9, 6 gait subphases of squat mode are realized by switched control algorithm shown in Table 2. The dynamic characteristics of 2 knee angles in squat mode are shown in Figure 18. The gait subphases are described by time slice $h \sim i(0 \mathrm{~s} \sim 2 \mathrm{~s}), i \sim j(2 \mathrm{~s} \sim$ $4 \mathrm{~s}), j \sim k(4 \mathrm{~s} \sim 7 \mathrm{~s}), k \sim l(7 \mathrm{~s} \sim 29 \mathrm{~s}), l \sim m(29 \mathrm{~s} \sim 32 \mathrm{~s})$, and $m \sim$ $n(32 \mathrm{~s} \sim 35 \mathrm{~s})$, respectively. In gait (5), described as time slice $h \sim i$, the double stance phase should be passed before the squat mode begins. The cylinder positions are controlled to reach the tester's stance posture shown in Figure 19. In gait (6), described as time slice $i \sim j$, the right leg swings to prepare the squat action and the tester regulates the center of gravity at this moment. In gait (7), described as time slice $j \sim k$, the left cylinder is retracted gradually by exponential fader control shown in Table 2. So the left leg bends gradually before the right knee contacts the ground. The control current is reduced from 0 to $-u_{\max }$ in $3 \mathrm{sec}$ shown in Figure 20. In gait (8), described as time slice $k \sim l, 2$ control currents are minus maximal value. The exoskeleton can not compensate its mass by 2 hydraulic actuators at this phase. The tester can bear the load himself through regulating the center of gravity to reduce the backward torque caused by exoskeleton mass and hold in the prescript posture. In gait (9), described as time slice $l \sim m, 2$ cylinders are extended to realize the up-squat action in $3 \mathrm{sec}$ (Figure 21). The cylinder positions of 2 legs are controlled by robust control algorithm to reach the suitable positions where gait (9) is switched into gait (2). After upsquat phase ends, the squat mode will return to the walking mode.

6.3. Experimental Scene. The experimental scenes of humanmachine coordinated motion are shown in Figures 22 and 23. In walking mode, the tester can bear the external load $60 \mathrm{~kg}$ hung on the back frame of exoskeleton. When the waking gait is switched, the tester will bear a little more load himself due to the regulation of center of gravity. In the stable stance phases, such as double standing or single leg stance, the exoskeleton can guarantee the aid-force effect by the proposed robust controller. Also, the comfortable effect of tester is improved by the switched control algorithm.

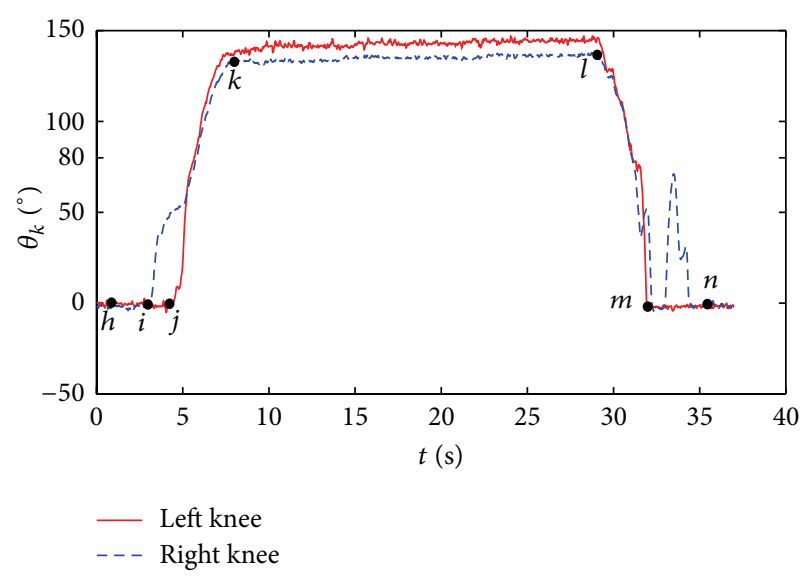

FIGURE 18: The knee angles of 2 legs in squat mode.

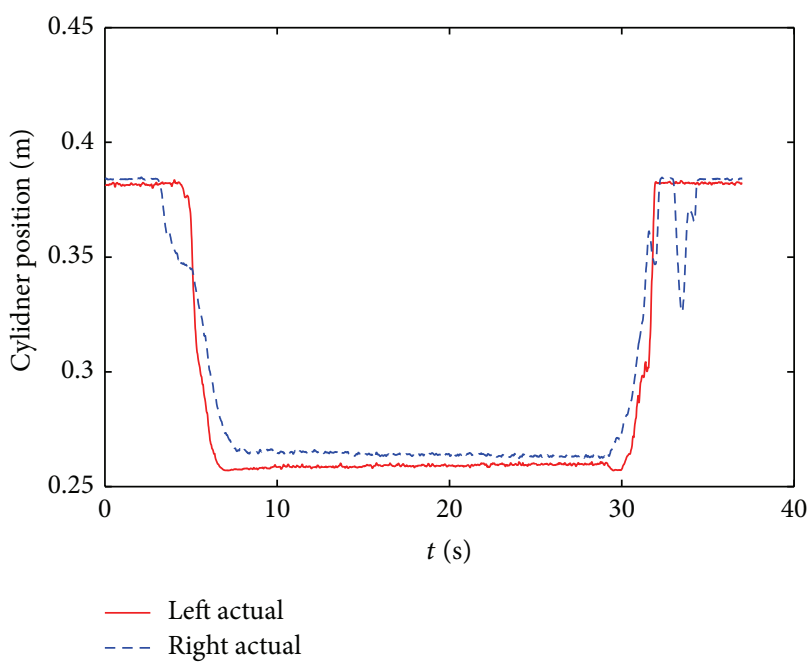

FIGURE 19: The cylinder position of 2 hydraulic actuators in squat mode.

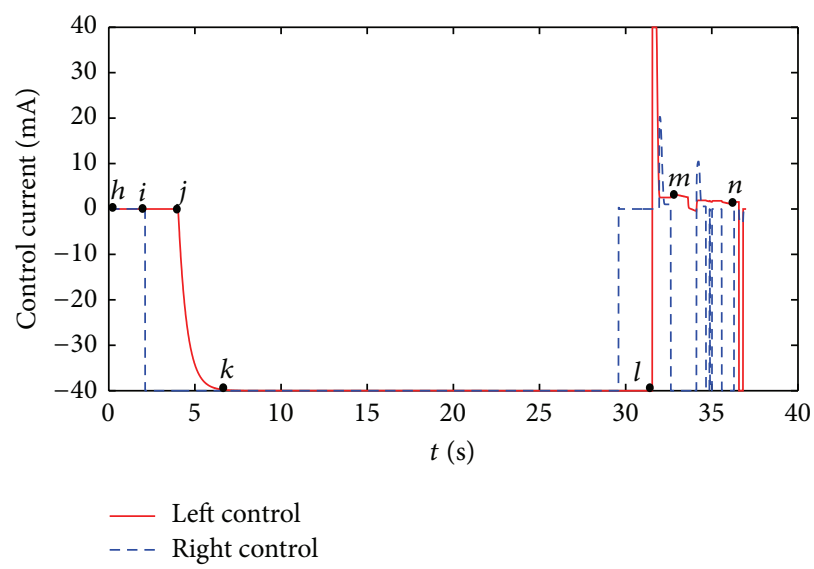

FIGURE 20: The control current of 2 servo valves in squat mode. 


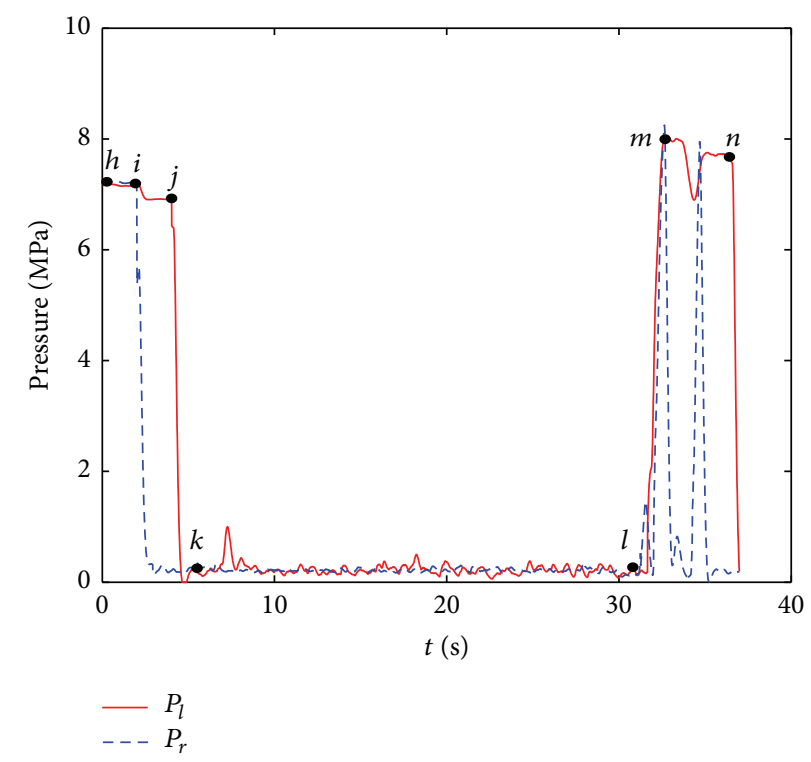

FIgURE 21: The pressures of 2 hydraulic actuators in squat mode.

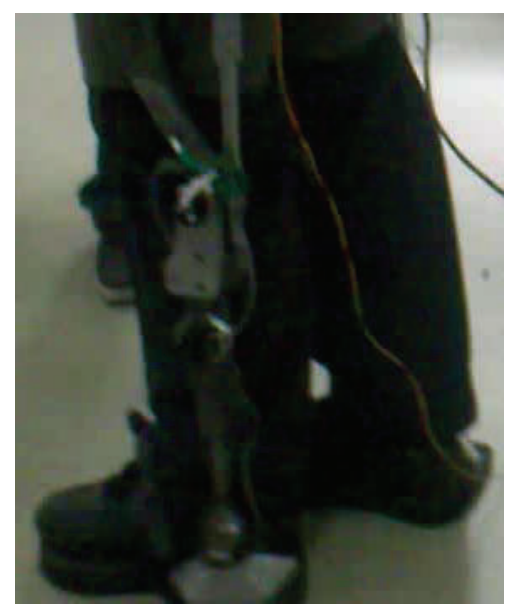

(a) Gait (1)

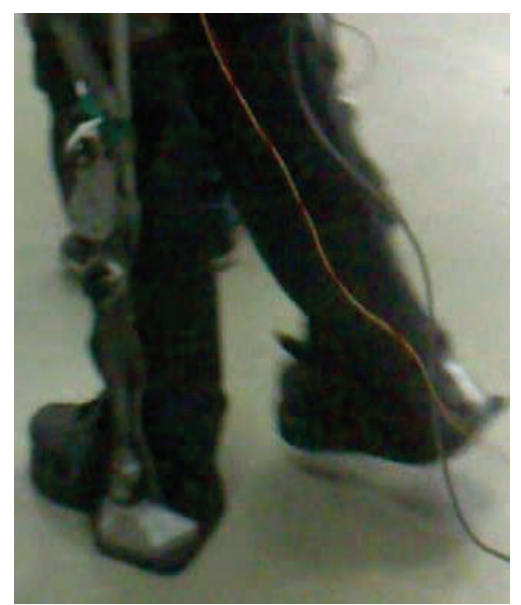

(b) Gait (2)

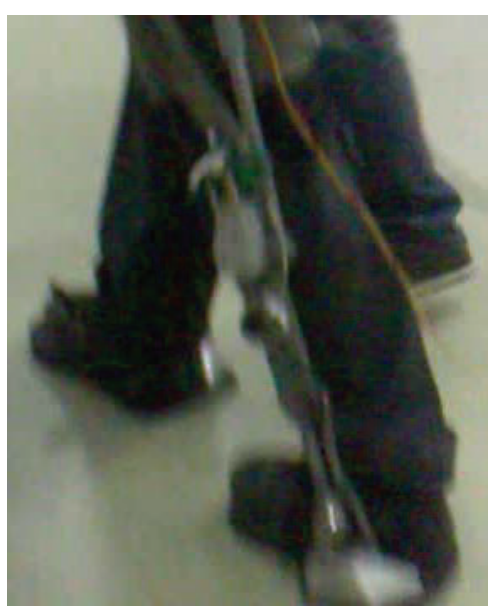

(c) Gait (3)

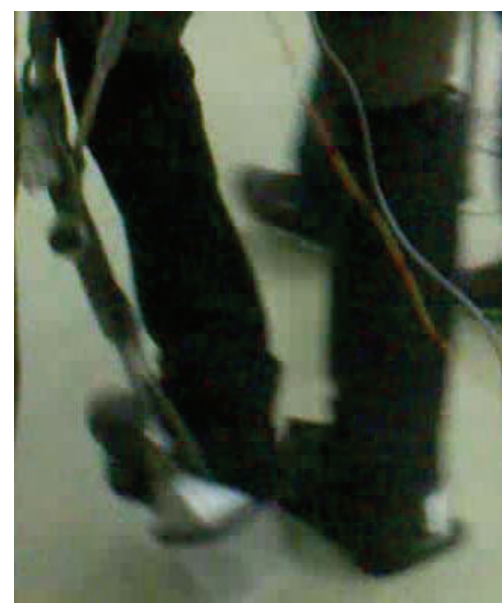

(d) Gait (4)

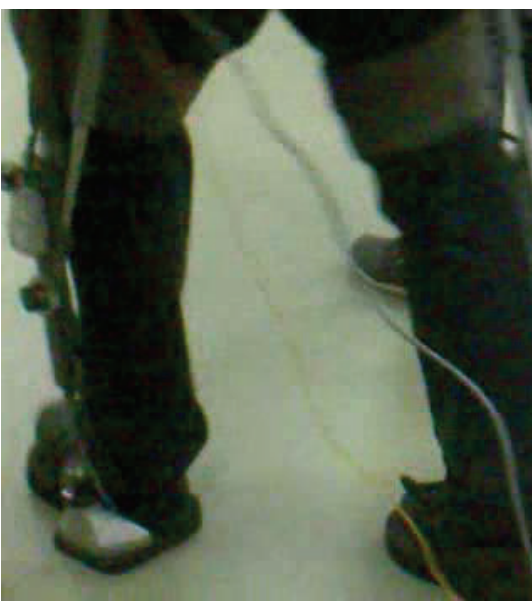

(e) Gait (5)

FIgURE 22: The walking gait subphases experiment. 


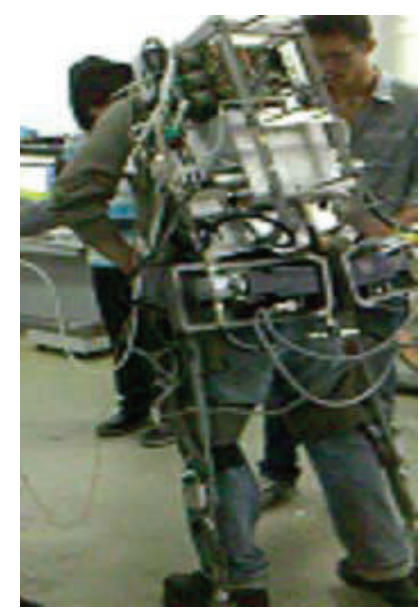

(a) Gait (6)

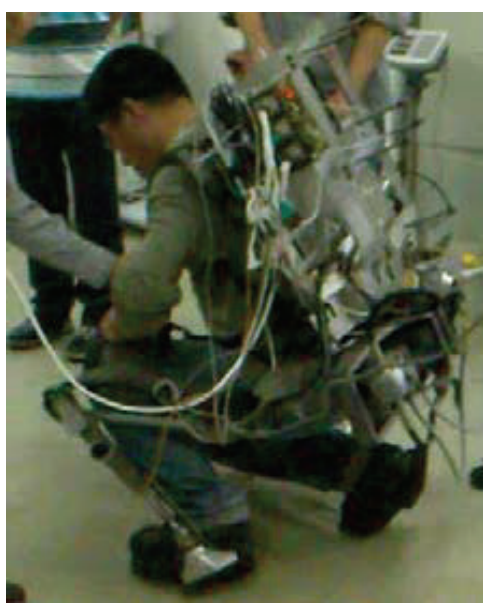

(c) Gait (8)

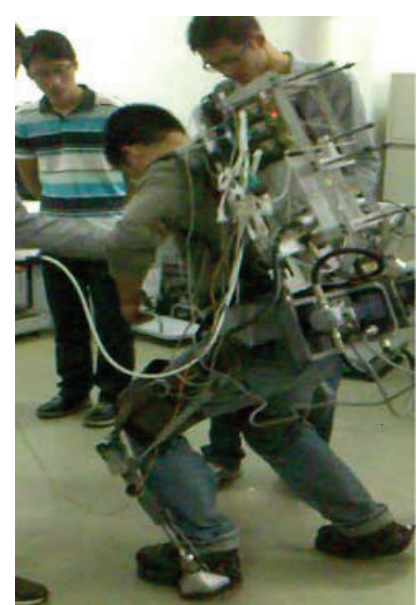

(b) Gait (7)

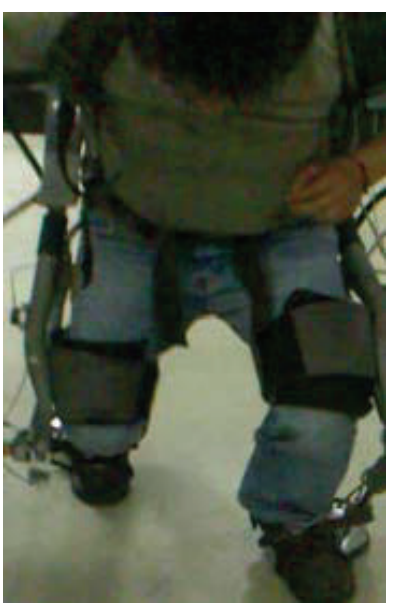

(d) Gait (9)

FIGURE 23: The squat gait subphases experiment.

In squat mode, the tester only bears the exoskeleton mass because of the safety of system at the present stage. The tester can realize the basic gait of squat action under the help of another person's arm, especially in gait (7) and gait (9).

\section{Conclusion}

In this paper, a human-machined coordinated motion control method is presented to realize the aid-force of lower extremity exoskeleton. This proposed method improves the robust performance under the structural and parametric uncertainty disturbance. The positions of 2 hydraulic actuators are controlled to make exoskeleton track person's motion by output feedback control. Compared with conventional PID control method in simulation, the proposed method can achieve a better dynamic response performance and aid-force effect in stance phase of walking gait. According to the gait divisions of human-machined coordinated motion, the switched control algorithm is designed to reduce the delay of exoskeleton tracking person. The experimental results of 2 motion modes validate the effectiveness of this proposed method and gait switched control algorithm.

\section{Conflict of Interests}

The authors declare that there is no conflict of interests regarding the publication of this paper.

\section{Acknowledgments}

This research was supported by project of National Natural Science Foundation of China (no. 61305092 and no. 51205045) and project of China Postdoctoral Science Foundation (no. 2013M542487). The authors would like to thank anonymous reviewers for their valuable comments and suggestions.

\section{References}

[1] S. Ackerman, The Report of Production Display for "HULC" Made in Lockheed Martin, Montgomery County, Md, USA, 2010, http://www.lockheedmartin.com.

[2] Z. X. Wang, "Raytheon launched XOS2 second-generation exoskeleton device," Light Weapons, pp. 24-44, 2010. 
[3] J. R. Steger, A design and control methodology for human exoskeletons [Ph.D. dissertation], Department of Mechanical Engineering, University of California, Berkeley, Calif, USA, 2006.

[4] J. F. Veneman, R. Ekkelenkamp, R. Kruidhof, F. C. T. van der Helm, and H. van der Kooij, "Design of a series elasticand Bowden cable-based actuation system for use as torqueactuator in exoskeleton-type training," in Proceedings of the IEEE 9th International Conference on Rehabilitation Robotics (ICORR '05), pp. 496-499, Chicago, Ill, USA, July 2005.

[5] Verge, "The report of 'Hercule exoskeleton can help a regular human carry up to 220 pounds"' 2012, http://www.theverge .com/2012/2/22/2815704/hercule-exoskeleton-human-carry220-pounds.

[6] C. G. Atkeson, J. G. Hale, F. Pollick et al., "Using humanoid robots to study human behavior," IEEE Intelligent Systems and Their Applications, vol. 15, no. 4, pp. 46-55, 2000.

[7] Q. Guo, H. Zhou, and D. Jiang, "Coordinated control method of the lower extremity exoskeleton based on human electromechanical coupling," in Social Robotics: 4th International Conference, ICSR 2012, Chengdu, China, October 29-31, 2012. Proceedings, vol. 7621 of Lecture Notes in Computer Science, pp. 650-659, Springer, Berlin, Germany, 2012.

[8] Q. Li, M. Kong, Z. Du, L. Sun, and D. Wang, "Interactive rehabilitation exercise control strategy for 5-DOF upper limb rehabilitation arm," Chinese Journal of Mechanical Engineering, vol. 44, no. 9, pp. 169-176, 2008.

[9] Z. Y. Yang, Y. S. Zhang, W. J. Gu et al., "Bone clothing sensitivity amplification control," Computer Simulation, vol. 27, no. 1, pp. 177-180, 2010.

[10] H. Cao, X. W. Meng, Z. Y. Ling et al., "Two-legged robot exoskeleton plantar pressure measurement system," Journ of Sensors and Actuators, vol. 23, no. 3, pp. 326-330, 2010.

[11] R. Fales and A. Kelkar, "Robust control design for a wheel loader using $H_{\infty}$ and feedback linearization based methods," ISA Transactions, vol. 48, no. 3, pp. 312-320, 2009.

[12] G. P. Liu and S. Daley, "Optimal-tuning PID controller design in the frequency domain with application to a rotary hydraulic system," Control Engineering Practice, vol. 7, no. 7, pp. 821-830, 1999.

[13] I. Boiko, "Variable-structure PID controller for level process," Control Engineering Practice, vol. 21, no. 5, pp. 700-707, 2013.

[14] M. Moradi, "Self-tuning PID controller to three-axis stabilization of a satellite with unknown parameters," International Journal of Non-Linear Mechanics, vol. 49, pp. 50-56, 2013.

[15] O. Cerman and P. Hušek, "Adaptive fuzzy sliding mode control for electro-hydraulic servo mechanism," Expert Systems with Applications, vol. 39, no. 11, pp. 10269-10277, 2012.

[16] C. Guan and S. X. Pan, "Adaptive sliding mode control of electro-hydraulic system with nonlinear unknown parameters," Control Engineering Practice, vol. 16, no. 11, pp. 1275-1284, 2008.

[17] D. H. Sha, V. B. Bajic, and H. Y. Yang, "New model and sliding mode control of hydraulic elevator velocity tracking system," Simulation Practice and Theory, vol. 9, no. 6-8, pp. 365-385, 2002.

[18] C. W. Wang, Z. X. Jiao, S. Wu, and Y. X. Shang, "Nonlinear adaptive torque control of electro-hydraulic load system with external active motion disturbance," Mechatronics, vol. 24, no. 1, pp. 32-40, 2014.
[19] I. Ursu, A. Toader, A. Halanay, and S. Balea, "New stabilization and tracking control laws for electrohydraulic servomechanisms," European Journal of Control, vol. 19, no. 1, pp. 65-80, 2013.

[20] V. Milić, Ž. Ŝitum, and M. Essert, "Robust $H_{\infty}$ position control synthesis of an electro-hydraulic servo system," ISA Transactions, vol. 49, no. 4, pp. 535-542, 2010.

[21] F. L. Weng, Y. C. Ding, and M. K. Tang, "LPV model-based robust controller design of electro-hydraulic servo systems," Procedia Engineering, vol. 15, pp. 421-425, 2011.

[22] X. Song, Y. Wang, and Z. Sun, "Robust stabilizer design for linear time-varying internal model based output regulation and its application to an electrohydraulic system," Automatica, vol. 50, no. 4, pp. 1128-1134, 2014.

[23] R. Fales and A. Kelkar, "Robust control design for a wheel loader using mixed sensitivity $\mathrm{H}$-infinity and feedback linearization based methods," in Proceedings of the American Control Conference (ACC '05), pp. 4381-4386, Portland, Ore, USA, June 2005.

[24] S. Kawamura and M. Svinin, Advances in Robot Control: From Everyday Physics to Human-like Movements, Springer, Berlin, Germany, 2006.

[25] A. Akers, M. Gassman, and R. Smith, Hydraulic Power System Analysis, Taylor \& Francis Group, 2006.

[26] A. Alleyne and R. Liu, "A simplified approach to force control for electro-hydraulic systems," Control Engineering Practice, vol. 8, no. 12, pp. 1347-1356, 2000.

[27] W.-M. Lu, K. M. Zhou, and J. C. Doyle, "Stabilization of uncertain linear systems: an LFT approach," IEEE Transactions on Automatic Control, vol. 41, no. 1, pp. 50-65, 1996.

[28] M. K. H. Fan, A. L. Tits, and J. C. Doyle, "Robustness in the presence of mixed parametric uncertainty and unmodeled dynamics," IEEE Transactions on Automatic Control, vol. 36, no. 1, pp. 25-38, 1991.

[29] D. W. Gu, P. H. Petkov, and M. M. Konstantinov, Robust control design with MATLAB, Springer Press, 2005.

[30] J. C. Doyle, K. Glover, P. P. Khargonekar, and B. A. Francis, "State-space solutions to standard $H_{2}$ and $H_{\infty}$ control problems," IEEE Transactions on Automatic Control, vol. 34, no. 8, pp. 831-847, 1989.

[31] G. J. Balas and J. C. Doyle, "Robustness and performance tradeoffs in control design for flexible structures," IEEE Transactions on Control Systems Technology, vol. 2, no. 4, pp. 352-361, 1994.

[32] A. Packard, M. K. H. Fan, and J. C. Doyle, "A power method for the structured singular value," in Proceedings of the 27th IEEE Conference on Decision and Control, pp. 2132-2137, Chicago, Ill, USA, December 1988.

[33] W. Gawronski, "Balanced systems and structures: reduction, assignment, and perturbations," in Control and Dynamic Systems, vol. 54, pp. 373-415, 1992. 


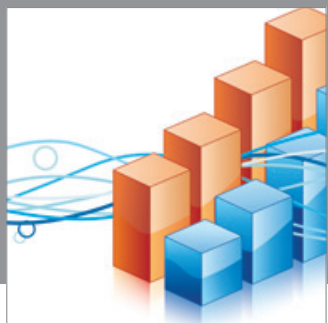

Advances in

Operations Research

mansans

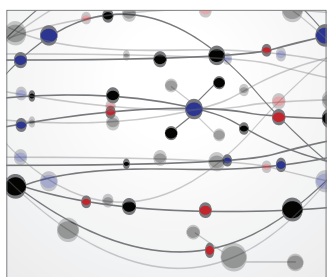

The Scientific World Journal
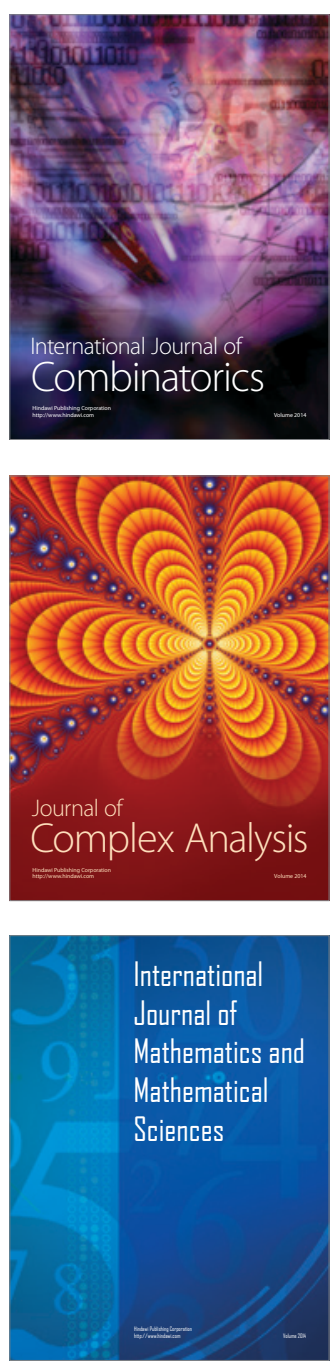
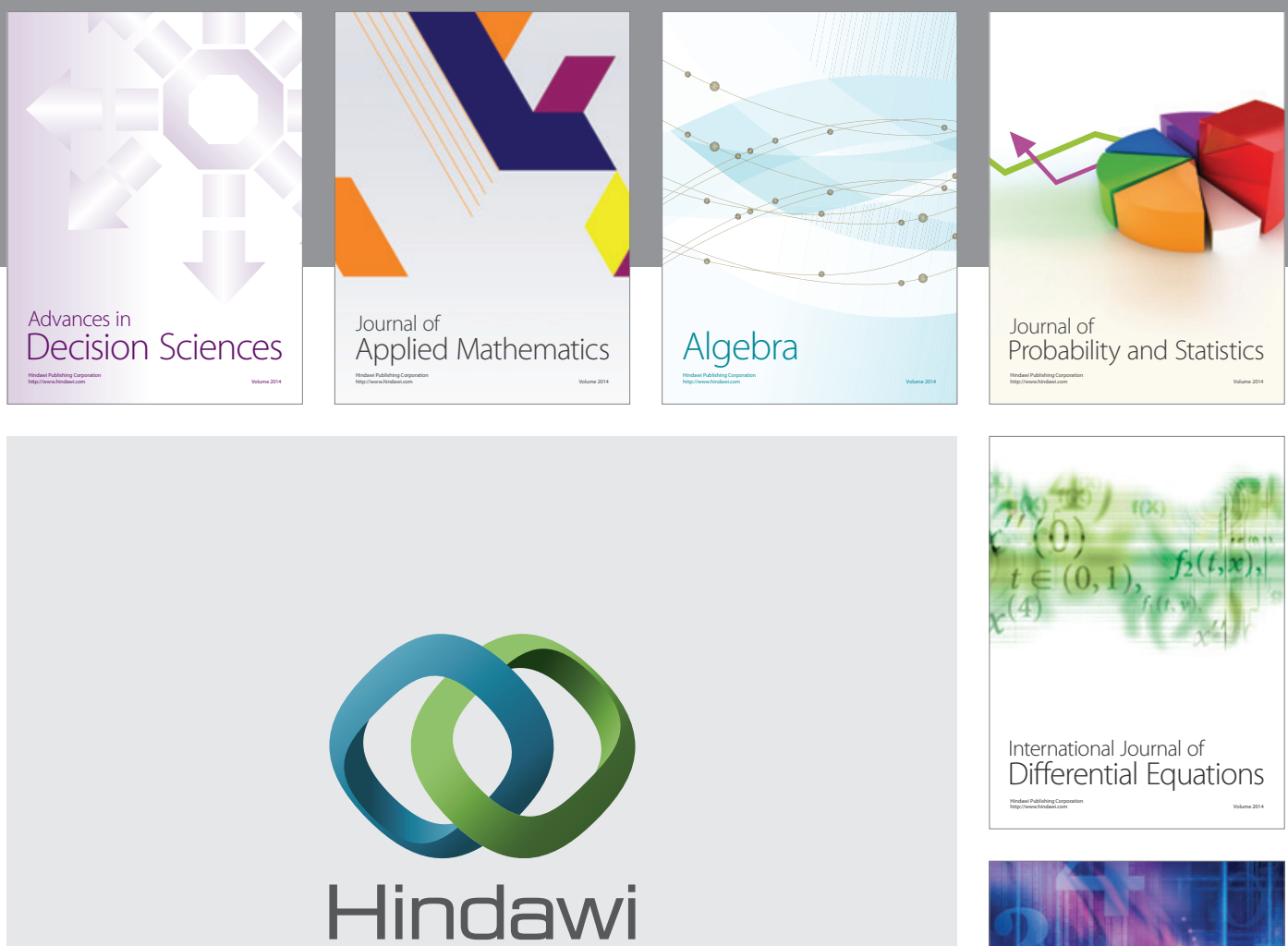

Submit your manuscripts at http://www.hindawi.com
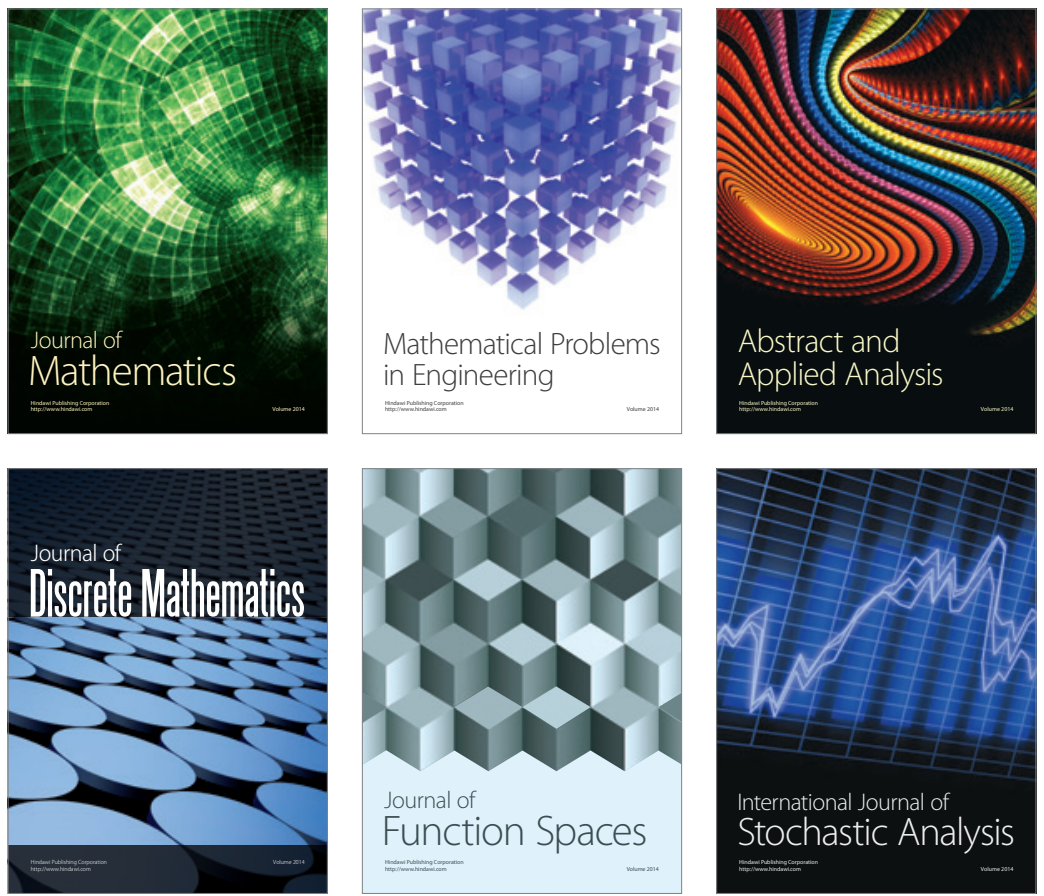

Journal of

Function Spaces

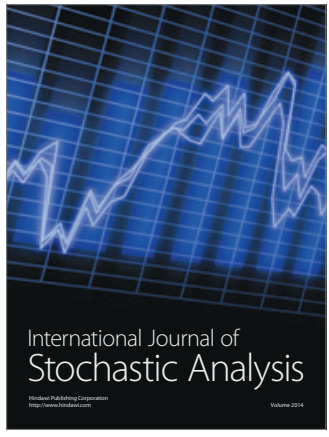

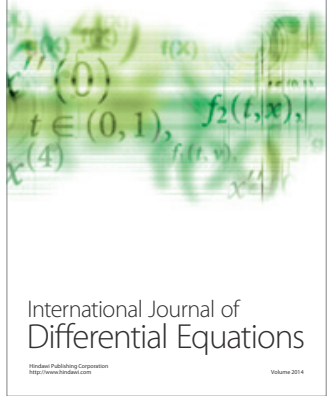
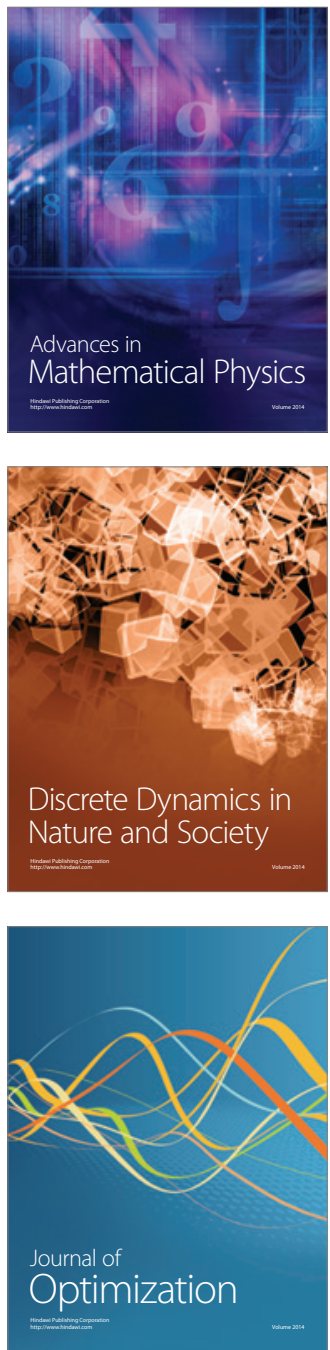